$11-1-2016$

\title{
A New Test for Correlation on Bivariate Nonnormal Distributions
}

\author{
Ping Wang \\ Great Basin College, ping.wang@gbcnv.edu \\ Ping Sa \\ University of North Florida, psa@unf.edu
}

Follow this and additional works at: http://digitalcommons.wayne.edu/jmasm

Part of the Applied Statistics Commons, Social and Behavioral Sciences Commons, and the $\underline{\text { Statistical Theory Commons }}$

\section{Recommended Citation}

Wang, Ping and Sa, Ping (2016) "A New Test for Correlation on Bivariate Nonnormal Distributions," Journal of Modern Applied Statistical Methods: Vol. 15 : Iss. 2 , Article 18.

DOI: $10.22237 / \mathrm{jmasm} / 1478002560$

Available at: http://digitalcommons.wayne.edu/jmasm/vol15/iss2/18

This Regular Article is brought to you for free and open access by the Open Access Journals at DigitalCommons@WayneState. It has been accepted for inclusion in Journal of Modern Applied Statistical Methods by an authorized editor of DigitalCommons@WayneState. 


\section{A New Test for Correlation on Bivariate Nonnormal Distributions}

Ping Wang

Great Basin College

Elko, NV
Ping Sa

University of North Florida

Jacksonville, FL

A new method to conduct a right-tailed test for the correlation on bivariate non-normal distribution is proposed. The comparative simulation study shows that the new test controls the type I error rates well for all the distributions considered. An investigation of the power performance is also provided.

Keywords: Correlation, Edgeworth expansion, Cornish-Fisher inverse expansion, type I error rate, power performance

\section{Introduction}

Bivariate data is data that has two variables. In the bivariate case, the study of the relationship between the two variables is at least as important as analyzing each variable individually. The most popular measure of the strength of the linear relation between two variables is the correlation coefficient, denoted by $\rho$. The Pearson product-moment correlation, $r$, is the most frequently-used estimator for $\rho$. Another widely-used estimator is the Spearman's rank correlation, denoted by $r_{s}$.

\section{Tests Based on Pearson Product-Moment Correlation}

Pearson (1896) developed the initial mathematical formulas for the sample correlation coefficient. Let $\left(X_{i}, Y_{i}\right), i=1, \ldots, n$ be a random sample, the statistic $r$ is given by:

$$
r=\frac{s_{X Y}}{s_{X} s_{Y}}=\frac{\sum\left(X_{i}-\bar{X}\right)\left(Y_{i}-\bar{Y}\right)}{\left[\sum\left(X_{i}-\bar{X}\right)^{2} \sum\left(Y_{i}-\bar{Y}\right)^{2}\right]^{1 / 2}}
$$

Ms. Wang is the Director of the Academic Success and Testing Center. Email her at: ping.wang@gbcnv.edu. Dr. Sa is a Professor in the Department of Mathematics and Statistics.Email herat: psa@unf.edu. 
where $s_{X Y}$ is the sample covariance of $X$ and $Y, n$ is the sample size, $s_{X}$ and $s_{Y}$ are the sample standard deviations, and $\bar{X}$ and $\bar{Y}$ are the sample means for the variables $X$ and $Y$, respectively.

The Pearson product-moment correlation $r$ is the maximum likelihood estimator of the parameter $\rho$ when the population has a bivariate normal distribution. Although $r$ is a biased estimator, the bias is negligible when the sample size is large. Researchers have done intensive work on the distribution of $r$ when the population is bivariate normal (Fisher, 1915; Stuart \& Ord, 1994).

$r$ can be used to test $\mathrm{H}_{0}: \rho=0$ when the population is a bivariate normal distribution. The test statistic

$$
t_{r}^{*}=r \sqrt{\frac{n-2}{1-r^{2}}}
$$

follows the Student's $t$-distribution with $n-2$ degrees of freedom under $\mathrm{H}_{0}$.

$r$ can also be used to test $\mathrm{H}_{0}: \rho=\rho_{0}$, for $-1 \leq \rho_{0} \leq 1$. The sampling distribution of $r$ is complicated and unstable even when the population is bivariate normal. Fisher (1921) introduced a remarkable transformation of $r$, which tends to normality much faster. When the sample size $n$ is moderately large, given

$$
r^{*}=\frac{1}{2} \ln \frac{1+r}{1-r}, \quad \rho^{*}=\frac{1}{2} \ln \frac{1+\rho}{1-\rho}
$$

the distribution of $r^{*}-\rho^{*}$ approaches to normal with an approximate mean $\rho / 2(n-1)$ and variance $1 / n-3$. Note that $n>50$ is an adequate sample size for the above approximation (see David, 1938).

To test $\mathrm{H}_{0}: \rho=\rho_{0}$, the test statistic is:

$$
z_{F}=\frac{\frac{1}{2} \ln \frac{1+r}{1-r}-\frac{1}{2} \ln \frac{1+\rho_{0}}{1-\rho_{0}}-\frac{\rho_{0}}{2(n-1)}}{\sqrt{\frac{1}{n-3}}}
$$

$z_{F}$ has approximately a standard normal distribution under $\mathrm{H}_{0}$. 


\section{A NEW TEST FOR CORRELATION}

\section{Test Based on Spearman Rank Correlation}

Spearman (1904) proposed a rank correlation which can be used to measure the relationship between two variables when the distribution is neither bivariate normal nor transformed to a bivariate normal. The Spearman rank correlation, $r_{s}$, is a nonparametric version of the Pearson product-moment correlation. Let $\left(R_{1 i}, R_{2 i}\right)$, $i=1, \ldots, n$ be the paired rank data of two variables, $r_{s}$ is given by:

$$
r_{s}=\frac{s_{R_{1} R_{2}}}{s_{R_{1}} S_{R_{2}}}=\frac{\sum\left(R_{1 i}-\bar{R}_{1}\right)\left(R_{2 i}-\bar{R}_{2}\right)}{\left[\sum\left(R_{1 i}-\bar{R}_{1}\right)^{2} \sum\left(R_{2 i}-\bar{R}_{2}\right)^{2}\right]^{1 / 2}}
$$

where $s_{R_{1} R_{2}}$ is the sample covariance of the paired ranks and $s_{R_{1}}$ and $\bar{R}_{1}, s_{R_{2}}$ and $\bar{R}_{2}$ are the sample standard deviation and the sample mean of the ranks of the two variables, respectively.

The Spearman rank correlation $r_{s}$ can be used to test:

$$
\mathrm{H}_{0} \text { : there is no association between the rank pairs }
$$

The test statistic is

$$
t_{s}^{*}=r_{s} \sqrt{\frac{n-2}{1-r_{s}^{2}}}
$$

which follows the Student's $t$-distribution with $n-2$ degrees of freedom under $\mathrm{H}_{0}$.

\section{Other Tests on Correlation}

The test based on $r$ can only be used when the population is bivariate normal or the sample size is relatively large. Although the test based on $r_{s}$ is applicable to the distribution-free case, it is less powerful and limited to test for zero correlation. However, in real world situations, most distributions are not bivariate normal and the sample sizes may not be large. Furthermore, a test of non-zero correlation is often required. It is desired to develop methods to meet these needs.

Beasley et al. (2007) proposed two new approaches to test a non-zero by using the bootstrapping method. Their methods do not require any knowledge of the population. One is the hypothesis-imposed univarite sampling bootstrap (HI) and the other one is the observed-imposed univariate sampling bootstrap (OI). Two tests 
are conducted on populations with various combinations of normal and skewed variates with $\rho \geq 0.4$ and the sample size $n \geq 10$. Their study demonstrated that although $\mathrm{OI}$ is preferable to $\mathrm{HI}$ under the significance level of 0.05 , the type I error rates are still slightly inflated. Also, the simulated populations are limited to the combinations between normal and skewed populations. The methods are not evaluated under the situations that both variables are non-normal. Another drawback of these two methods is that they are computer-intensive methods. Unfortunately, most practitioners do not have the computer programming skills to implement these methods.

Beversdorf and Sa (2011) proposed tests of correlation for bivariate nonnormal data with small sample sizes. The tests investigated are Fisher's Z transformation $z_{F}$ and the saddlepoint approximation $r_{L}$. They found that $z_{F}$ and $r_{L}$ have extremely similar performance which could control the type I error rates well when a left-tailed test was performed under all the bivariate non-normal distributions considered. Both methods essentially failed to control the type I error rates when a right-tailed test is desired.

The purpose of this study is to develop a new right-tailed test on bivariate non-normal distributions with non-zero correlation. The new test statistic is derived using the Edgeworth expansion and the Cornish-Fisher inverse expansion.

\section{Methodology}

\section{Edgeworth Expansion}

The Edgeworth expansion was derived by Edgeworth (1905), and uses a series to approximate a probability distribution in terms of its cumulants. Let $\hat{\theta}$ be an estimator of an unknown parameter $\theta$, and $\sqrt{n}(\hat{\theta}-\theta)$ be asymptotically normally distributed with mean zero and variance $\sigma^{2}$. Hall (1983) developed the Edgeworth expansion of the distribution function of $\sqrt{n}(\hat{\theta}-\theta)$ as a power series in $\sqrt{n}$.

$$
\mathrm{P}\left\{\frac{\sqrt{n}(\hat{\theta}-\theta)}{\sigma} \leq u\right\}=\Phi(u)+n^{-1 / 2} \mathrm{p}_{1}(u) \phi(u)+\ldots+n^{-j / 2} \mathrm{p}_{j}(u) \phi(u)+\ldots
$$




\section{A NEW TEST FOR CORRELATION}

where $\Phi(u), \phi(u)$, and $\mathrm{p}_{j}(u)$ denote the standard normal distribution function, its density function, and a polynomial function with coefficients depending on cumulants of $\hat{\theta}-\theta$, respectively.

The inverse of the Edgeworth expansion, obtained by inverting the formula (2), is known as the Cornish-Fisher expansion:

$$
\mathrm{P}\left\{\frac{\sqrt{n}(\hat{\theta}-\theta)}{\sigma} \leq z+n^{-1 / 2} \mathrm{p}_{11}(z)+n^{-1} \mathrm{p}_{21}(z)+\ldots+n^{-j / 2} \mathrm{p}_{j 1}(z)+\ldots\right\}=\Phi(z)
$$

where $z$ is the percentile of the standard normal distribution and the $\mathrm{p}_{j 1}$ are polynomials defined in terms of $\mathrm{p}_{j} \mathrm{~s}$ (Hall, 1992).

\section{Proposed Test Procedure}

Assume that a bivariate population has finite cumulants and a correlation coefficient $\rho$. Let $\kappa_{01}, \kappa_{10}, \kappa_{02}, \kappa_{20}, \kappa_{11}, \ldots$ up to order six be the product cumulants for the bivariate population. Then $r_{*}=\sqrt{n}(r-\rho)$ has a limiting normal distribution with mean zero and constant variance $\sigma^{2}$, where $\sigma^{2}$ is of the form (Nakagawa \& Niki, 1992):

$$
\begin{gathered}
\sigma^{2}=\frac{1}{\kappa_{20} \kappa_{02}}\left(\frac{1}{4} \kappa_{40} \kappa_{20}^{-2} \kappa_{11}^{2}-\kappa_{31} \kappa_{20}^{-1} \kappa_{11}+\frac{1}{2} \kappa_{22} \kappa_{20}^{-1} \kappa_{11}^{2} \kappa_{02}^{-1}+\kappa_{22}+\kappa_{20} \kappa_{02}+\kappa_{20}^{-1} \kappa_{11}^{4} \kappa_{02}^{-1}\right. \\
\left.-\kappa_{13} \kappa_{11} \kappa_{02}^{-1}+\frac{1}{4} \kappa_{11}^{2} \kappa_{04} \kappa_{02}^{-2}-2 \kappa_{11}^{2}\right)
\end{gathered}
$$

Bhattacharya and Ghosh (1978) provided the Edgeworth expansion of $R$, where

$$
R=\frac{r_{*}}{\sigma}=\frac{\sqrt{n}(r-\rho)}{\sigma}
$$

as 


$$
\begin{aligned}
\mathrm{P}(R<u)=\Phi(u)-\phi(u) & {\left[\frac{1}{\sqrt{n}}\left\{\frac{1}{6} \frac{v_{3}}{\sigma^{3}} \mathrm{H}_{2}(u)+\frac{v_{1}}{\sigma}\right\}+\frac{1}{n}\left\{\frac{1}{72} \frac{v_{3}^{2}}{\sigma^{6}} \mathrm{H}_{5}(u)\right.\right.} \\
+ & \left(\frac{1}{6} \frac{v_{1} v_{3}}{\sigma^{4}}+\frac{1}{24} \frac{v_{4}}{\sigma^{4}} \mathrm{H}_{3}(u)\right) \\
+ & \left.\left.\left(\frac{1}{2} \frac{v_{2}}{\sigma^{2}}+\frac{1}{2} \frac{v_{2}}{\sigma^{2}}\right) \mathrm{H}_{1}(u)\right\}\right]+\mathrm{O}\left(\frac{1}{n \sqrt{n}}\right)
\end{aligned}
$$

where $\Phi(u)$ and $\phi(u)$ denote the standard normal distribution function and its density function, $\mathrm{O}(1 / n \sqrt{n})$ is the big-oh function of order $1 / n \sqrt{n}, \mathrm{H}_{1}(u), \mathrm{H}_{2}(u)$, $\mathrm{H}_{3}(u)$, and $\mathrm{H}_{5}(u)$ are Hermite polynomials with

$$
\mathrm{H}_{1}(u)=u ; \quad \mathrm{H}_{2}(u)=u^{2}-1 ; \quad \mathrm{H}_{3}(u)=u^{3}-3 u ; \quad \mathrm{H}_{5}(u)=u^{5}-10 u^{3}+15 u,
$$

and $v_{1}, v_{2}, v_{3}$, and $v_{4}$ are parameters such that

$$
\frac{1}{\sqrt{n}} \frac{v_{1}}{\sigma}, \quad 1+\frac{1}{n} \frac{v_{2}}{\sigma^{2}}, \quad \frac{1}{\sqrt{n}} \frac{v_{3}}{\sigma^{3}}, \quad \frac{1}{n} \frac{v_{4}}{\sigma^{4}}
$$

are the approximate cumulants of $R$. The explicit forms of $v_{1}, v_{2}, v_{3}$, and $v_{4}$ were provided by Nakagawa and Niki (1992). Formulas for calculating $v_{1}$ and $v_{3}$ are listed in Appendix A. Formulas for calculating $v_{2}$ and $v_{4}$ are not needed in this study.

Nakagawa and Niki (1992) applied the inverted Edgeworth expansion to the distribution of $R$ of order $1 / n$ :

$$
\begin{aligned}
\mathrm{P}(R \leq & z+\frac{1}{\sqrt{n}}\left\{\frac{1}{6} \frac{v_{3}}{\sigma^{3}}\left(z^{2}-1\right)+\frac{v_{1}}{\sigma}\right\}+\frac{1}{n}\left\{z^{3}\left(-\frac{1}{18} \frac{v_{3}^{2}}{\sigma^{6}}+\frac{1}{24} \frac{v_{4}}{\sigma^{4}}\right)\right. \\
& \left.\left.+z\left(\frac{1}{2} \frac{v_{2}}{\sigma^{2}}+\frac{5}{36} \frac{v_{3}^{2}}{\sigma^{6}}-\frac{1}{8} \frac{v_{4}}{\sigma^{4}}\right)\right\}\right) \\
& =\Phi(z)+\mathrm{O}\left(\frac{1}{n \sqrt{n}}\right)
\end{aligned}
$$

If only order $1 / \sqrt{n}$ is required, then (4) can be reduced to a simpler form: 


\section{A NEW TEST FOR CORRELATION}

$$
\mathrm{P}\left(R \leq z+B_{1}\right)=\Phi(z)+\mathrm{O}\left(\frac{1}{n}\right)
$$

where $B_{1}=\frac{1}{\sqrt{n}}\left\{\frac{1}{6} \frac{v_{3}}{\sigma^{3}}\left(z^{2}-1\right)+\frac{v_{1}}{\sigma}\right\}$.

To test $\mathrm{H}_{0}: \rho=\rho_{0}$ versus $\mathrm{H}_{\mathrm{a}}: \rho>\rho_{0}$, the intuitive decision rule is:

reject $\mathrm{H}_{0}: \rho=\rho_{0}$ when $R>z+B_{1}$, i.e.

reject $\mathrm{H}_{0}: \rho=\rho_{0}$ under the significance level $\alpha$, if

$$
\frac{\sqrt{n}\left(r-\rho_{0}\right)}{\sigma}>z_{\alpha}+\frac{1}{\sqrt{n}}\left\{\frac{1}{6} \frac{v_{3}}{\sigma^{3}}\left(z_{\alpha}^{2}-1\right)+\frac{v_{1}}{\sigma}\right\}
$$

Since negative values of $B_{1}$ might increase type I errors, the following adjustment is proposed: Define

$$
B_{2}=\max \left(z_{\alpha}, z_{\alpha}+\frac{1}{\sqrt{n}}\left\{\frac{1}{6} \frac{v_{3}}{\sigma^{3}}\left(z_{\alpha}^{2}-1\right)+\frac{v_{1}}{\sigma}\right\}\right)
$$

The decision rule is adjusted to:

$$
\text { reject } \mathrm{H}_{0} \text { if } \frac{\sqrt{n}\left(r-\rho_{0}\right)}{\sigma}>B_{2}
$$

All the parameters in (6) and (7) can be written in terms of the product cumulants. These product cumulants $\kappa_{i j}$ are estimated by their corresponding unbiased estimators $k_{i j}$. Detailed formulas are provided in Appendix B.

For the special case of $\rho_{0}=0, \kappa_{01}=\kappa_{10}=0, \kappa_{02}=\kappa_{20}=1$, and $\kappa_{p q}=0$ for $p+q \geq 5$, Nakagawa and Niki (1992) gave the simplified forms for parameters $\sigma^{2}$, $v_{1}$, and $v_{3}$ as follows:

$$
\begin{aligned}
& \sigma^{2}=1+\kappa_{22} \\
& v_{1}=-\frac{1}{2}\left(\kappa_{13}+\kappa_{31}\right) \\
& v_{3}=3 \kappa_{12} \kappa_{21}+\kappa_{03} \kappa_{30}-3 \kappa_{22}\left(\kappa_{13}+\kappa_{31}\right)
\end{aligned}
$$


To test $\mathrm{H}_{0}: \rho=0$, (6) and (7) are evaluated with the parameters given in (9). Again, all the parameters are estimated by their corresponding unbiased estimators.

\section{Simulation Study}

The simulation study was implemented to evaluate type I error rates, to investigate the power performance, and to compare with the existing Fisher's Z transformation method on the type I error rates.

\section{Simulation Description}

Fleishman (1978) proposed a method to generate univariate non-normal random variables with desired coefficients of skewness $\beta$ and kurtosis $\gamma$. Vale and Maurelli (1983) extended Fleishman's method to the bivariate non-normal case with a specified correlation coefficient. Five parameters, including two sets of skewness and kurtosis and one correlation coefficient, are required to generate the bivariate non-normal data using Vale and Maurelli method.

Seven levels of the skewness, $-3.0,-1.2,-0.5,0.0,0.5,1.2$, and 3.0, and five levels of the kurtosis, 0.0, 4.0, 10.0, 14.0, and 25.0, were considered, and 24 combinations were selected. Moreover, five correlation coefficients, 0.0, 0.5, 0.6, 0.75 , and 0.9 , three significance levels, $0.10,0.05$, and 0.01 , and two sample sizes, 15 and 30, were used in the simulation study.

Two new methods and the Fisher's $Z$ transformation method were evaluated. The method using (6) was denoted by Zb, and the one using (8) was denoted by Zc. The Fisher's Z transformation method (1) was shortened as Zf. Both Zb and Zc methods were evaluated with two critical values, $z_{\alpha}$ and $t_{(\alpha, n-2)}$.

\section{The Algorithm of the Test on Correlation:}

1. Input the desired $\rho_{X, Y}$ and two sets of skewness and kurtosis, $\left(\beta_{1}, \gamma_{1}\right)$ and $\left(\beta_{2}, \gamma_{2}\right)$.

2. Generate $n$ bivariate non-normal random variates $(X, Y)$ based on the given parameters.

3. Calculate $z_{F}$ in (1), Zb in (6), and $\mathrm{Zc}$ in (8).

4. Compare the tests with their critical values; count one if the test is rejected.

5. Repeat (2) - (4) 99,999 times. 


\section{A NEW TEST FOR CORRELATION}

6. Calculate the type I error rate, the proportion of the false rejection (out of $100,000)$ for each test.

In the power study, an extra parameter $\rho_{a}$ is input in step (1) and used to generate the data as the true population correlation. However, all of the test statistics in step (3) are evaluated under $\rho_{0}$. All fo the simulations were run with Fortran 77 for Windows on an IBM T61 Laptop Computer.

\section{Simulation Results}

\section{Type I Error Rate Comparisons}

Tables 1-3 provide the comparative study of the type I error rates on various bivariate non-normal distributions with significance levels $0.10,0.05$, and 0.01 and sample size 30. Comparisons were made among the tests $\mathrm{Zf}, \mathrm{Zb}$, and $\mathrm{Zc}$ with two critical values, $z_{\alpha}$ and $t_{(\alpha, n-2)}$, while $Z$ f only used the critical value, $z_{\alpha}$. The correlation coefficients $0.00,0.50,0.60,0.75$, and 0.90 were targeted during the simulation study. A total of 24 bivariate non-normal distributions with various population conditions were examined.

Table 1 shows the results on testing a zero correlation. It can be observed that the $\mathrm{Zc}$ method controls the type I error rates well. On the contrary, the $\mathrm{Zb}$ method do not control type I error rates at all. Almost all of the type I error rates obtained by the $\mathrm{Zb}$ method are slightly inflated except for a few cases. The Zf method can control the type I error rates as long as the skewness and kurtosis are small. Once theses parameters increase, Zf becomes unstable and fails to control the type I error rates in many cases.

More specifically, in testing $\rho_{0}=0$ on a distribution which is bivariate normal or very close to bivariate normal, Zf controls type I error rates a bit better than Zc. However, for the non-normal distributions, $\mathrm{Zc}$ is better than $\mathrm{Zf}$ in controlling type I error rates.

Tables 2 and 3 give the results for right-tailed tests on the non-zero correlation. It is quite interesting to see that the hypothesized value $\rho_{0}$ actually affects the type I error rate performance. When $n=30$ and $\rho_{0}=0.5$, both the $\mathrm{Zf}$ and $\mathrm{Zb}$ methods basically fail to control the type I error rates with very few exceptions. The type I error rates obtained by the Zc method have better performance. However, the cases with controlled type I error rates are restricted to the distributions with small to moderate skewness and kurtosis. When $\rho_{0}$ increases to 0.6, the $\mathrm{Zc}$ method successfully controls the type I error rates for nearly all the distributions considered 


\section{WANG \& SA}

with the $t$ critical point. As $\rho_{0}$ increases, the type I error rates get more conservative. This tendency can be observed on both $\mathrm{Zb}$ and $\mathrm{Zc}$ methods.

The Zf method fails completely in the right-tailed test on non-zero correlation with only a few exceptions. This result confirms with the study by Beversdorf and $\mathrm{Sa}$ (2011). Their study shows that Zf can properly control the type I error rates on the left-tailed test but not on the right-tailed test. Therefore, it is fair to conclude that, for the right-tailed test, the only method that can properly control the type I error rates is the $\mathrm{Zc}$ method with the $t$ critical point.

Due to the similar results in the study, only the moderate sample size 30 and significance levels of 0.05 and 0.01 are reported in the tables.

\section{Power Results}

The power performance of the proposed test is also evaluated. Tables 4 and 5 provide the power performance to test $\rho_{0}=0$ when $\rho_{a}=0.0,0.2,0.4,0.6$, and 0.8 with significance levels 0.05 and 0.01 . Table 6 provides a small-scale investigation on the power performance to test $\rho_{0}=0.55$ and $\rho_{a}=0.6$ and 0.7 .

Both the Zf and Zc methods perform well in testing $\rho_{0}=0$. In testing on an exactly- or nearly-normal distribution, the power from $\mathrm{Zf}$ and $\mathrm{Zc}$ converges to 1 quickly. When $\rho_{a}=0.6$, both achieve a power of 0.99 ; when $\rho_{a}=0.8$, the power rates are essentially 1 . For the distributions with large skewness and kurtosis, the Zc method, which is the only one with controlled type I error rates, converges to 1 more slowly but still reasonably well. A small-scale power study to test non-zero correlation is presented in Table 6. At significance level 0.10 , sample size 30, $\rho_{0}=0.55$ versus $\rho_{a}=0.6$ and 0.7 , it is observed that the power of Zc steadily increases when $\rho_{a}$ moves away from $\rho_{0}$.

\section{Conclusions}

This study proposed a new right-tailed test for the correlation of bivariate nonnormal distributions. This new test adapts the inverse Edgeworth expansion for the standardized correlation $R=\frac{\sqrt{n}(r-\rho)}{\sigma}$ by Nakagawa and Niki (1992).

This newly proposed test can be conducted without any knowledge of the populations. The simulation study shows that this new right-tailed test has the best performance in controlling the type I error rates. The proposed method, along with the $t$ critical point, can be used to test both $\rho_{0}=0$ and any value of $\rho_{0}$ when $\rho_{0}>0.5$.

The power performance of the new test was also evaluated. $\mathrm{Zc}$ is as powerful as Zf when testing $\rho_{0}=0$. To test non-zero correlations, it is meaningless to 


\section{A NEW TEST FOR CORRELATION}

compare the two tests since Zf fails to control type I error rates. The power examination of the Zc method shows that the power steadily goes up when $\rho_{a}$ moves away from $\rho_{0}$.

The new test does have its own limitations. It cannot control the type I error rates well when the population has a small correlation and it is a right-tailed test. In order to better control the type I error rates, a higher-order Edgeworth expansion may be considered. Unfortunately, this might lead to tedious computations when higher-order terms are introduced in the test.

\section{References}

Beasley, W. H., DeShea, L., Toothaker, L. E., Mendoza, J. L., Bard, D. E., \& Rodgers, J. L. (2007). Bootstrapping to test for nonzero population correlation coefficients using univariate sampling. Psychological Methods, 12(4), 414-433. doi: 10.1037/1082-989X.12.4.414

Beversdorf, L. M., \& Sa, P. (2011). Tests for correlation on bivariate nonnormal distributions. Journal of Modern Applied Statistical Methods, 10(2), 699709. Retrieved from http://digitalcommons. wayne.edu/jmasm/vol10/iss2/29/

Bhattacharya, R. N., \& Ghosh, J. K. (1978). On the validity of the formal Edgeworth expansion. The Annals of Statistics, 6(2), 434-451. Available from http://www.jstor.org/stable/2958887

David, F. N. (1938). Tables of the correlation coefficient. London: Cambridge University Press.

Edgeworth, F. Y. (1905). The law of error. Transactions of the Cambridge Philosophical Society, 20, 36-65, 113-141.

Fisher, R. A. (1915). Frequency distribution of the values of the correlation coefficient in samples from an indefinitely large population. Biometrika, 10(4), 507-521. doi: 10.2307/2331838

Fisher, R. A. (1921). On the "probable error" of a coefficient of correlation deduced from a small sample. Metron, 1, 3-32.

Fisher, R. A. (1930). Moments and product moments of sampling distributions. Proceedings of the London Mathematical Society, s2-30(1), 199238. doi: 10.1112/plms/s2-30.1.199

Fleishman, A. I. (1978). A method of simulating non-normal distributions. Psychometrika, 43(4), 521-531. doi: 10.1007/BF02293811

Hall, P. (1983). Inverting an Edgeworth expansion. The Annals of Statistics, 11(2), 569-576. doi: 10.1214/aos/1176346162 
Hall, P. (1992). The bootstrap and Edgeworth expansion. New York, NY: Springer-Verlag.

Nakagawa, S., \& Niki, N. (1992). Distribution of sample correlation coefficient for nonnormal populations. Journal of the Japanese Society of Computational Statistics, 5(1), 1-19. doi: 10.5183/jjscs1988.5.1

Pearson, K. (1896). Mathematical contributions to the theory of evolution. III. Regression, heredity, and panmixia. Philosophical Transactions of the Royal Society of London. Series A, 187, 253-318. Available from http://www.jstor.org/stable/90707

Spearman, C. (1904). "General intelligence," Objectively determined and measured. The American Journal of Psychology, 15(2), 201-293. doi:

$10.2307 / 1412107$

Stuart, A., \& Ord, J. K. (1994). Kendall's advanced theory of statistics (6th ed.) (Vol. 1). New York, NY: Halsted Press.

Vale, D. C., \& Maurelli, V. A. (1983). Simulating multivariate non-normal distributions. Psychometrika, 48(3), 465-471. doi: 10.1007/BF02293687 


\section{A NEW TEST FOR CORRELATION}

\section{Appendix A: Formulae Used in Edgeworth Expansion of $\boldsymbol{R}$}

Let $\eta=\frac{1}{\sqrt{\kappa_{20} \kappa_{02}}}$. Then

$$
\begin{aligned}
& v_{1}=\eta\left(\frac{3}{8} \kappa_{40} \kappa_{20}^{-2} \kappa_{11}-\frac{1}{2} \kappa_{31} \kappa_{20}^{-1}+\frac{1}{4} \kappa_{22} \kappa_{20}^{-1} \kappa_{11} \kappa_{02}^{-1}+\frac{1}{2} \kappa_{20}^{-1} \kappa_{11}^{3} \kappa_{02}^{-1}-\frac{1}{2} \kappa_{13} \kappa_{02}^{-1}\right. \\
& \left.+\frac{3}{8} \kappa_{11} \kappa_{04} \kappa_{02}^{-2}-\frac{1}{2} \kappa_{11}\right) \\
& v_{3}=\eta^{3}\left(-\frac{1}{8} \kappa_{60} \kappa_{20}^{-3} \kappa_{11}^{3}+\frac{3}{4} \kappa_{51} \kappa_{20}^{-2} \kappa_{11}^{2}-\frac{3}{2} \kappa_{42} \kappa_{20}^{-1} \kappa_{11}-\frac{3}{8} \kappa_{42} \kappa_{20}^{-2} \kappa_{11}^{3} \kappa_{02}^{-1}+\frac{9}{16} \kappa_{40}^{2} \kappa_{20}^{-4} \kappa_{11}^{3}\right. \\
& -3 \kappa_{40} \kappa_{31} \kappa_{20}^{-3} \kappa_{11}^{2}+\frac{3}{2} \kappa_{40} \kappa_{22} \kappa_{20}^{-2} \kappa_{11}+\frac{3}{2} \kappa_{40} \kappa_{22} \kappa_{20}^{-3} \kappa_{11}^{3} \kappa_{02}^{-1}-\frac{3}{2} \kappa_{40} \kappa_{20}^{-2} \kappa_{13} \kappa_{11}^{2} \kappa_{02}^{-1} \\
& +\frac{3}{8} \kappa_{40} \kappa_{20}^{-2} \kappa_{11}^{3} \kappa_{04} \kappa_{02}^{-2}-3 \kappa_{40} \kappa_{20}^{-2} \kappa_{11}^{3}+3 \kappa_{40} \kappa_{20}^{-3} \kappa_{11}^{5} \kappa_{02}^{-1}+\frac{3}{2} \kappa_{33} \kappa_{20}^{-1} \kappa_{11}^{2} \kappa_{02}^{-1}+\kappa_{33} \\
& +\frac{15}{4} \kappa_{31}^{2} \kappa_{20}^{-2} \kappa_{11}-3 \kappa_{31} \kappa_{22} \kappa_{20}^{-1}-\frac{9}{2} \kappa_{31} \kappa_{22} \kappa_{20}^{-2} \kappa_{11}^{2} \kappa_{02}^{-1}+\frac{9}{2} \kappa_{31} \kappa_{20}^{-1} \kappa_{13} \kappa_{11} \kappa_{02}^{-1} \\
& -\frac{3}{2} \kappa_{31} \kappa_{20}^{-1} \kappa_{11}^{2} \kappa_{04} \kappa_{02}^{-2}+12 \kappa_{31} \kappa_{20}^{-1} \kappa_{11}^{2}-12 \kappa_{31} \kappa_{20}^{-2} \kappa_{11}^{4} \kappa_{02}^{-1}-\frac{1}{2} \kappa_{30}^{2} \kappa_{20}^{-3} \kappa_{11}^{3} \\
& +3 \kappa_{30} \kappa_{21} \kappa_{20}^{-2} \kappa_{11}^{2}-3 \kappa_{30} \kappa_{20}^{-1} \kappa_{12} \kappa_{11}+\kappa_{30} \kappa_{03}-\frac{3}{8} \kappa_{24} \kappa_{20}^{-1} \kappa_{11}^{3} \kappa_{02}^{-2}-\frac{3}{2} \kappa_{24} \kappa_{11} \kappa_{02}^{-1} \\
& +3 \kappa_{22}^{2} \kappa_{20}^{-1} \kappa_{11} \kappa_{02}^{-1}+\frac{3}{2} \kappa_{22}^{2} \kappa_{20}^{-2} \kappa_{11}^{3} \kappa_{02}^{-2}-\frac{9}{2} \kappa_{22} \kappa_{20}^{-1} \kappa_{13} \kappa_{11}^{2} \kappa_{02}^{-2}+\frac{3}{2} \kappa_{22} \kappa_{20}^{-1} \kappa_{11}^{3} \kappa_{04} \kappa_{02}^{-3} \\
& +6 \kappa_{22} \kappa_{20}^{-1} \kappa_{11}^{3} \kappa_{02}^{-1}+6 \kappa_{22} \kappa_{20}^{-2} \kappa_{11}^{5} \kappa_{02}^{-2}-3 \kappa_{22} \kappa_{13} \kappa_{02}^{-1}+\frac{3}{2} \kappa_{22} \kappa_{11} \kappa_{04} \kappa_{02}^{-2}-12 \kappa_{22} \kappa_{11} \\
& -3 \kappa_{21}^{2} \kappa_{20}^{-1} \kappa_{11}-\frac{3}{2} \kappa_{21}^{2} \kappa_{20}^{-2} \kappa_{11}^{3} \kappa_{02}^{-1}+6 \kappa_{21} \kappa_{20}^{-1} \kappa_{12} \kappa_{11}^{2} \kappa_{02}^{-1}+3 \kappa_{21} \kappa_{12}-3 \kappa_{21} \kappa_{11} \kappa_{03} \kappa_{02}^{-1} \\
& -6 \kappa_{20} \kappa_{11} \kappa_{02}-12 \kappa_{20}^{-1} \kappa_{13} \kappa_{11}^{4} \kappa_{02}^{-2}-\frac{3}{2} \kappa_{20}^{-1} \kappa_{12}^{2} \kappa_{11}^{3} \kappa_{02}^{-2}+3 \kappa_{20}^{-1} \kappa_{11}^{5} \kappa_{04} \kappa_{02}^{-3}-18 \kappa_{20}^{-1} \kappa_{11}^{5} \kappa_{02}^{-1} \\
& +6 \kappa_{20}^{-2} \kappa_{11}^{7} \kappa_{02}^{-2}+\frac{3}{4} \kappa_{15} \kappa_{11}^{2} \kappa_{02}^{-2}+\frac{15}{4} \kappa_{13}^{2} \kappa_{11} \kappa_{02}^{-2}-3 \kappa_{13} \kappa_{11}^{2} \kappa_{04} \kappa_{02}^{-3}+12 \kappa_{13} \kappa_{11}^{2} \kappa_{02}^{-1} \\
& -3 \kappa_{12}^{2} \kappa_{11} \kappa_{02}^{-1}+3 \kappa_{12} \kappa_{11}^{2} \kappa_{03} \kappa_{02}^{-2}-\frac{1}{8} \kappa_{11}^{3} \kappa_{06} \kappa_{02}^{-3}+\frac{9}{16} \kappa_{11}^{3} \kappa_{04}^{2} \kappa_{02}^{-4}-3 \kappa_{11}^{3} \kappa_{04} \kappa_{02}^{-2} \\
& \left.-\frac{1}{2} \kappa_{11}^{3} \kappa_{03}^{2} \kappa_{02}^{-3}+18 \kappa_{11}^{3}\right)
\end{aligned}
$$




\section{Appendix B: $k$-Statistics}

Fisher (1930) introduced $k$-statistics as the unbiased estimator of the $m^{\text {th }}$ cumulant $\kappa_{m}$, i.e. $\mathrm{E}\left(k_{m}\right)=\kappa_{m}$. Define the power sum of a univariate data as: $s_{m}=\sum_{i=1}^{n} x_{i}^{m}$, the first six $k$-statistics in terms of the corresponding $\kappa_{m}$ are ( See Stuart \& Ord, 1994):

$$
\begin{aligned}
& k_{1}=\frac{1}{n^{[1]}} s_{1}, \quad k_{2}=\frac{1}{n^{[2]}}\left(n s_{2}-s_{1}^{2}\right), \\
& k_{3}=\frac{1}{n^{[3]}}\left(n^{2} s_{3}-3 n s_{2} s_{1}+2 s_{1}^{3}\right), \\
& k_{4}=\frac{1}{n^{[4]}}\left\{\left(n^{3}+n^{2}\right) s_{4}-4\left(n^{2}+n\right) s_{3} s_{1}-3\left(n^{2}-n\right) s_{2}^{2}+12 n s_{2} s_{1}^{2}-6 s_{1}^{4}\right\}, \\
& k_{5}=\frac{1}{n^{[5]}}\left\{\left(n^{4}+5 n^{3}\right) s_{5}-5\left(n^{3}+5 n^{2}\right) s_{4} s_{1}-10\left(n^{3}-n^{2}\right) s_{3} s_{2}+20\left(n^{2}+2 n\right) s_{3} s_{1}^{2}\right. \\
&\left.\quad+30\left(n^{2}-n\right) s_{2}^{2} s_{1}-60 n s_{2} s_{1}^{3}+24 s_{1}^{5}\right\}, \\
& k_{6}=\frac{1}{n^{[6]}}\left\{\left(n^{5}+16 n^{4}+11 n^{3}-4 n^{2}\right) s_{6}-6\left(n^{4}+16 n^{3}+11 n^{2}-4 n\right) s_{5} s_{1}\right. \\
& \quad-15 n(n-1)^{2}(n+4) s_{4} s_{2}-10\left(n^{4}-2 n^{3}+5 n^{2}-4 n\right) s_{3}^{2} \\
& \quad+30\left(n^{3}+9 n^{2}+2 n\right) s_{4} s_{1}^{2}+120\left(n^{3}-n\right) s_{3} s_{2} s_{1}+30\left(n^{3}-3 n^{2}+2 n\right) s_{2}^{3} \\
&\left.\quad-120\left(n^{2}+3 n\right) s_{3} s_{1}^{3}-270\left(n^{2}-n\right) s_{2}^{2} s_{1}^{2}+360 n s_{2} s_{1}^{4}-120 s_{1}^{6}\right\} \\
&\left.\quad+30 \ell_{7} s_{2}^{3}-120 \ell_{8} s_{3} s_{1}^{2}-270 \ell_{9} s_{2}^{2} s_{1}^{2}+360 \ell_{10} s_{2} s_{1}^{4}-120 s_{1}^{6}\right\} \\
& \frac{1}{n^{[6]}}\left\{\ell_{1} s_{6}-6 \ell_{2} s_{5} s_{1}-15 \ell_{3} s_{4} s_{2}-10 \ell_{4} s_{3}^{2}+30 \ell_{5} s_{4} s_{1}^{2}+120 \ell_{6} s_{3} s_{2} s_{1}\right. \\
& \quad
\end{aligned}
$$

Stuart and Ord (1994) also provided an approach to derive the multivariate $k$ statistics. Define $s_{r t}=\sum_{i=1}^{n} x_{i}^{r} y_{i}^{t}$, where $\left(x_{i}, y_{i}\right), i=1,2, \ldots, n$ are the bivariate random observations. The following multivariate $k$-statistics can be derived:

$$
\begin{aligned}
& k_{11}=\frac{1}{n^{[2]}}\left(n s_{11}-s_{10} s_{01}\right) \\
& k_{21}=\frac{1}{n^{[3]}}\left(n^{2} s_{21}-2 n s_{11} s_{10}-n s_{20} s_{01}+2 s_{10}^{2} s_{01}\right)
\end{aligned}
$$




\section{A NEW TEST FOR CORRELATION}

$$
\begin{aligned}
& k_{12}=\frac{1}{n^{[3]}}\left(n^{2} s_{12}-2 n s_{11} s_{01}-n s_{02} s_{10}+2 s_{01}^{2} s_{10}\right) \\
& k_{31}=\left\{\frac{1}{n^{[4]}}\left(n^{3}+n^{2}\right) s_{31}-3\left(n^{2}+n\right) s_{21} s_{10}-\left(n^{2}+n\right) s_{30} s_{01}-3\left(n^{2}-n\right) s_{20} s_{11}\right. \\
& \left.+6 n s_{11} s_{10}^{2}+6 n s_{20} s_{10} s_{01}-6 s_{10}^{3} s_{01}\right\} \\
& k_{13}=\left\{\frac{1}{n^{[4]}}\left(n^{3}+n^{2}\right) s_{13}-3\left(n^{2}+n\right) s_{12} s_{01}-\left(n^{2}+n\right) s_{03} s_{10}-3\left(n^{2}-n\right) s_{02} s_{11}\right. \\
& \left.+6 n s_{11} s_{01}^{2}+6 n s_{02} s_{01} s_{10}-6 s_{01}^{3} s_{10}\right\} \\
& k_{22}=\left\{\frac{1}{n^{[4]}}\left(n^{3}+n^{2}\right) s_{22}-2\left(n^{2}+n\right) s_{21} s_{01}-2\left(n^{2}+n\right) s_{12} s_{10}-2\left(n^{2}-n\right) s_{11}^{2}\right. \\
& \left.-\left(n^{2}-n\right) s_{20} s_{02}+2 n s_{02} s_{10}^{2}+8 n s_{11} s_{10} s_{01}+2 n s_{20} s_{01}^{2}-6 s_{10}^{2} s_{01}^{2}\right\} \\
& k_{51}=\frac{1}{n^{[6]}}\left\{\ell_{1} s_{51}-5 \ell_{2} s_{41} s_{10}-\ell_{2} s_{50} s_{01}-10 \ell_{3} s_{31} s_{20}-5 \ell_{3} s_{40} s_{11}-10 \ell_{4} s_{30} s_{21}\right. \\
& +20 \ell_{5} s_{31} s_{10}^{2}+10 \ell_{5} s_{40} s_{10} s_{01}+60 \ell_{6} s_{21} s_{20} s_{10}+40 \ell_{6} s_{30} s_{11} s_{10} \\
& +20 \ell_{6} s_{30} s_{20} s_{01}+30 \ell_{7} s_{20}^{2} s_{11}-60 \ell_{8} s_{21} s_{10}^{3}-60 \ell_{8} s_{30} s_{10}^{2} s_{01} \\
& -180 \ell_{9} s_{20} s_{11} s_{10}^{2}-90 \ell_{9} s_{20}^{2} s_{10} s_{01}+120 \ell_{10} s_{11} s_{10}^{4} \\
& \left.+240 \ell_{10} s_{20} s_{10}^{3} s_{01}-120 s_{10}^{5} s_{01}\right\} \\
& k_{42}=\frac{1}{n^{[6]}}\left\{\ell_{1} s_{42}-4 \ell_{2} s_{32} s_{10}-2 \ell_{2} s_{41} s_{01}-6 \ell_{3} s_{22} s_{20}-8 \ell_{3} s_{31} s_{11}-\ell_{3} s_{40} s_{02}\right. \\
& -6 \ell_{4} s_{21}^{2}-4 \ell_{4} s_{30} s_{12}+12 \ell_{5} s_{22} s_{10}^{2}+16 \ell_{5} s_{31} s_{10} s_{01}+2 \ell_{5} s_{40} s_{01}^{2} \\
& +24 \ell_{6} s_{12} s_{20} s_{10}+12 \ell_{6} s_{21} s_{20} s_{01}+48 \ell_{6} s_{21} s_{11} s_{10}+8 \ell_{6} s_{30} s_{02} s_{10} \\
& +12 \ell_{6} s_{21} s_{20} s_{01}+16 \ell_{6} s_{30} s_{11} s_{01}+12 \ell_{7} s_{20} s_{11}^{2}+6 \ell_{7} s_{20}^{2} s_{02} \\
& -24 \ell_{8} s_{12} s_{10}^{3}-72 \ell_{8} s_{21} s_{10}^{2} s_{01}-24 \ell_{8} s_{30} s_{10} s_{01}^{2}-72 \ell_{9} s_{11}^{2} s_{10}^{2} \\
& -36 \ell_{9} s_{20} s_{02} s_{10}^{2}-144 \ell_{9} s_{20} s_{11} s_{10} s_{01}-18 \ell_{9} s_{20}^{2} s_{01}^{2}+24 \ell_{10} s_{02} s_{10}^{4} \\
& \left.+192 \ell_{10} s_{11} s_{10}^{3} s_{01}+144 \ell_{10} s_{20} s_{10}^{2} s_{01}^{2}-120 s_{10}^{4} s_{01}^{2}\right\}
\end{aligned}
$$




\section{WANG \& SA}

$$
\begin{aligned}
& k_{24}=\frac{1}{n^{[6]}}\left\{\ell_{1} s_{24}-4 \ell_{2} s_{23} s_{01}-2 \ell_{2} s_{14} s_{10}-6 \ell_{3} s_{22} s_{02}-8 \ell_{3} s_{13} s_{11}-\ell_{3} s_{04} s_{20}\right. \\
&-6 \ell_{4} s_{12}^{2}-4 \ell_{4} s_{03} s_{21}+12 \ell_{5} s_{22} s_{01}^{2}+16 \ell_{5} s_{13} s_{01} s_{10}+2 \ell_{5} s_{04} s_{10}^{2} \\
&+24 \ell_{6} s_{21} s_{02} s_{01}+12 \ell_{6} s_{12} s_{02} s_{10}+48 \ell_{6} s_{12} s_{11} s_{01}+8 \ell_{6} s_{03} s_{20} s_{01} \\
&+12 \ell_{6} s_{12} s_{02} s_{10}+16 \ell_{6} s_{03} s_{11} s_{10}+12 \ell_{7} s_{02} s_{11}^{2}+6 \ell_{7} s_{02}^{2} s_{20} \\
&-24 \ell_{8} s_{21} s_{01}^{3}-72 \ell_{8} s_{12} s_{01}^{2} s_{10}-24 \ell_{8} s_{03} s_{01} s_{10}^{2}-72 \ell_{9} s_{11}^{2} s_{01}^{2} \\
&-36 \ell_{9} s_{02} s_{20} s_{01}^{2}-144 \ell_{9} s_{02} s_{11} s_{01} s_{10}-18 \ell_{9} s_{02}^{2} s_{10}^{2}+24 \ell_{10} s_{20} s_{01}^{4} \\
&\left.+192 \ell_{10} s_{11} s_{01}^{3} s_{10}+144 \ell_{10} s_{02} s_{01}^{2} s_{10}^{2}-120 s_{01}^{4} s_{10}^{2}\right\} \\
& 1+\ell_{1} s_{33}-3 \ell_{2} s_{23} s_{10}-3 \ell_{2} s_{32} s_{01}-3 \ell_{3} s_{13} s_{20}-3 \ell_{3} s_{31} s_{02}-9 \ell_{3} s_{22} s_{11} \\
& k_{33}=\frac{6}{6]}-9 \ell_{4} s_{21} s_{12}-\ell_{4} s_{30} s_{03}+6 \ell_{5} s_{13} s_{10}^{2}+18 \ell_{5} s_{22} s_{10} s_{01}+6 \ell_{5} s_{31} s_{01}^{2} \\
&+6 \ell_{6} s_{20} s_{10} s_{03}+36 \ell_{6} s_{12} s_{11} s_{10}+18 \ell_{6} s_{12} s_{20} s_{01}+36 \ell_{6} s_{21} s_{11} s_{01} \\
&+18 \ell_{6} s_{21} s_{10} s_{02}+6 \ell_{6} s_{30} s_{01} s_{02}+6 \ell_{7} s_{11}^{3}+12 \ell_{7} s_{20} s_{11} s_{02} \\
&-6 \ell_{8} s_{10}^{3} s_{03}-54 \ell_{8} s_{12} s_{10}^{2} s_{01}-54 \ell_{8} s_{21} s_{10} s_{01}^{2}-6 \ell_{8} s_{30} s_{01}^{3} \\
&-54 \ell_{9} s_{11} s_{10}^{2} s_{02}-108 \ell_{9} s_{11}^{2} s_{10} s_{01}-54 \ell_{9} s_{20} s_{10} s_{01} s_{02} \\
&-54 \ell_{9} s_{20} s_{11} s_{01}^{2}+72 \ell_{10} s_{10}^{3} s_{02} s_{01}+72 \ell_{10} s_{20} s_{10} s_{01}^{3} \\
&\left.+216 \ell_{10} s_{10}^{2} s_{01}^{2} s_{11}-120 s_{10}^{3} s_{01}^{3}\right\}
\end{aligned}
$$

where

$$
\begin{array}{ll}
n^{[1]}=n & n^{[2]}=n(n-1) \\
n^{[3]}=n(n-1)(n-2) & n^{[4]}=n(n-1)(n-2)(n-3) \\
n^{[5]}=n(n-1)(n-2)(n-3)(n-4) & \\
n^{[6]}=n(n-1)(n-2)(n-3)(n-4)(n-5) &
\end{array}
$$

and 


\section{A NEW TEST FOR CORRELATION}

$$
\begin{array}{ll}
\ell_{1}=n^{5}+16 n^{4}+11 n^{3}-4 n^{2} & \ell_{2}=n^{4}+16 n^{3}+11 n^{2}-4 n \\
\ell_{3}=n^{4}+2 n^{3}-7 n^{2}+4 n & \ell_{4}=n^{4}-2 n^{3}+5 n^{2}-4 n \\
\ell_{5}=n^{3}+9 n^{2}+2 n & \ell_{6}=n^{3}-n \\
\ell_{7}=n^{3}-3 n^{2}+2 n & \ell_{8}=n^{2}+3 n \\
\ell_{9}=n^{2}-n & \ell_{10}=n
\end{array}
$$




\section{Appendix C: Tables}

2 Table 1. Comparison of type I error rates $\left(\rho_{0}=0, n=30\right)$

\begin{tabular}{|c|c|c|c|c|c|c|c|c|c|c|c|}
\hline \multirow[b]{2}{*}{$\beta$} & \multirow[b]{2}{*}{$Y$} & \multirow[b]{2}{*}{$\mathrm{CP}$} & \multicolumn{3}{|c|}{$\alpha=0.10$} & \multicolumn{3}{|c|}{$\alpha=0.05$} & \multicolumn{3}{|c|}{$\alpha=0.01$} \\
\hline & & & $Z f$ & $\mathbf{Z b}$ & Zc & $\mathrm{Zf}$ & $\mathbf{Z b}$ & Zc & $\mathrm{Zf}$ & $\mathrm{Zb}$ & Zc \\
\hline 0.0 & 0 & $Z_{\alpha}$ & 0.0985 & 0.1171 & 0.1124 & 0.0491 & 0.0679 & 0.0632 & 0.0102 & 0.0262 & 0.0191 \\
\hline 0.0 & 0 & $t_{\alpha}$ & & 0.1124 & 0.1079 & & 0.0628 & 0.0580 & & 0.0227 & 0.0153 \\
\hline 0.0 & 1 & $z_{\alpha}$ & 0.0987 & 0.1268 & 0.1153 & 0.0510 & 0.0755 & 0.0640 & 0.0101 & 0.0306 & 0.0173 \\
\hline 0.0 & 1 & $t_{\alpha}$ & & 0.1213 & 0.1095 & & 0.0697 & 0.0579 & & 0.0273 & 0.0137 \\
\hline 0.0 & 10 & $Z_{\alpha}$ & 0.1034 & 0.1512 & 0.1061 & 0.0549 & 0.0958 & 0.0476 & 0.0144 & 0.0588 & 0.0083 \\
\hline 0.0 & 10 & $t_{\alpha}$ & & 0.1453 & 0.0998 & & 0.0900 & 0.0413 & & 0.0570 & 0.0057 \\
\hline 0.5 & 0 & $Z_{\alpha}$ & 0.0988 & 0.1118 & 0.1051 & 0.0508 & 0.0637 & 0.0569 & 0.0110 & 0.0232 & 0.0163 \\
\hline 0.5 & 0 & $t_{\alpha}$ & & 0.1064 & 0.1021 & & 0.0578 & 0.0541 & & 0.0195 & 0.0140 \\
\hline 1.0 & 0 & $Z \alpha$ & 0.1015 & 0.1014 & 0.0954 & 0.0559 & 0.0541 & 0.0499 & 0.0131 & 0.0164 & 0.0126 \\
\hline 1.0 & 0 & $t_{\alpha}$ & & 0.0959 & 0.0901 & & 0.0488 & 0.0446 & & 0.0138 & 0.0098 \\
\hline 0.5 & 1 & $Z \alpha$ & 0.0999 & 0.1225 & 0.1117 & 0.0516 & 0.0709 & 0.0604 & 0.0111 & 0.0275 & 0.0162 \\
\hline 0.5 & 1 & $t_{\alpha}$ & & 0.1168 & 0.1063 & & 0.0649 & 0.0548 & & 0.0242 & 0.0128 \\
\hline 1.2 & 4 & $Z_{\alpha}$ & 0.1055 & 0.1265 & 0.1015 & 0.0562 & 0.0734 & 0.0495 & 0.0143 & 0.0330 & 0.0104 \\
\hline 1.2 & 4 & $t_{\alpha}$ & & 0.1209 & 0.0958 & & 0.0674 & 0.0435 & & 0.0304 & 0.0075 \\
\hline 1.2 & 10 & $Z a$ & 0.1048 & 0.1433 & 0.1022 & 0.0580 & 0.0927 & 0.0469 & 0.0156 & 0.0539 & 0.0078 \\
\hline 1.2 & 10 & $t_{\alpha}$ & & 0.1380 & 0.0963 & & 0.0871 & 0.0412 & & 0.0522 & 0.0056 \\
\hline
\end{tabular}

5 Note: $\beta$ : skewness; $\gamma$ : kurtosis; CP: Critical Point 
A NEW TEST FOR CORRELATION

1 Table 1 (continued). Comparison of type I error rates $\left(\rho_{0}=0, n=30\right)$

\begin{tabular}{|c|c|c|c|c|c|c|c|c|c|c|c|}
\hline \multirow[b]{2}{*}{$\beta$} & \multirow[b]{2}{*}{$y$} & \multirow[b]{2}{*}{ CP } & \multicolumn{3}{|c|}{$\alpha=0.10$} & \multicolumn{3}{|c|}{$\alpha=0.05$} & \multicolumn{3}{|c|}{$\alpha=0.01$} \\
\hline & & & $Z f$ & $\mathrm{Zb}$ & Zc & $Z f$ & $\mathrm{Zb}$ & Zc & $Z f$ & $Z b$ & Zc \\
\hline 1.2 & 10 & $Z_{\alpha}$ & 0.1048 & 0.1433 & 0.1022 & 0.0580 & 0.0927 & 0.0469 & 0.0156 & 0.0539 & 0.0078 \\
\hline 1.2 & 10 & $t_{\alpha}$ & & 0.1380 & 0.0963 & & 0.0871 & 0.0412 & & 0.0522 & 0.0056 \\
\hline 1.2 & 25 & $Z_{\alpha}$ & 0.1026 & 0.1508 & 0.0913 & 0.0593 & 0.1022 & 0.0361 & 0.0214 & 0.0745 & 0.0046 \\
\hline 1.2 & 25 & $t_{\alpha}$ & & 0.1456 & 0.0852 & & 0.0975 & 0.0304 & & 0.0734 & 0.0031 \\
\hline-1.2 & 4 & $Z_{\alpha}$ & 0.1050 & 0.1262 & 0.1029 & 0.0561 & 0.0720 & 0.0482 & 0.0146 & 0.0322 & 0.0103 \\
\hline-1.2 & 4 & $t_{\alpha}$ & & 0.1208 & 0.0972 & & 0.0663 & 0.0429 & & 0.0297 & 0.0076 \\
\hline-1.2 & 10 & $Z_{\alpha}$ & 0.1051 & 0.1446 & 0.1021 & 0.0563 & 0.0915 & 0.0456 & 0.0159 & 0.0543 & 0.0075 \\
\hline-1.2 & 10 & $t_{\alpha}$ & & 0.1386 & 0.0960 & & 0.0853 & 0.0393 & & 0.0525 & 0.0054 \\
\hline-1.2 & 25 & $Z_{\alpha}$ & 0.1024 & 0.1507 & 0.0920 & 0.0589 & 0.1030 & 0.0358 & 0.0205 & 0.0739 & 0.0047 \\
\hline-1.2 & 25 & $t_{\alpha}$ & & 0.1450 & 0.0855 & & 0.0978 & 0.0303 & & 0.0730 & 0.0033 \\
\hline 1.2 & 4 & $Z_{\alpha}$ & 0.1057 & 0.1375 & 0.1043 & 0.0579 & 0.0860 & 0.0490 & 0.0145 & 0.0436 & 0.0090 \\
\hline 1.2 & 10 & $t_{\alpha}$ & & 0.1315 & 0.0984 & & 0.0797 & 0.0429 & & 0.0416 & 0.0067 \\
\hline 1.2 & 4 & $Z_{\alpha}$ & 0.1064 & 0.1498 & 0.1012 & 0.0562 & 0.0947 & 0.0429 & 0.0155 & 0.0599 & 0.0065 \\
\hline 1.2 & 25 & $t_{\alpha}$ & & 0.1437 & 0.0950 & & 0.0888 & 0.0373 & & 0.0583 & 0.0045 \\
\hline-1.2 & 4 & $Z_{\alpha}$ & 0.1047 & 0.1374 & 0.1032 & 0.0563 & 0.0841 & 0.0475 & 0.0143 & 0.0446 & 0.0087 \\
\hline-1.2 & 10 & $t_{\alpha}$ & & 0.1313 & 0.0970 & & 0.0781 & 0.0418 & & 0.0425 & 0.0063 \\
\hline-1.2 & 4 & $Z \alpha$ & 0.1052 & 0.1477 & 0.1004 & 0.0575 & 0.0968 & 0.0437 & 0.0162 & 0.0590 & 0.0066 \\
\hline-1.2 & 25 & $t_{\alpha}$ & & 0.1421 & 0.0940 & & 0.0908 & 0.0377 & & 0.0576 & 0.0050 \\
\hline
\end{tabular}

4 Note: $\beta$ : skewness; $\gamma$ : kurtosis; CP: Critical Point 


\section{WANG \& SA}

1 Table 1 (continued). Comparison of type I error rates $\left(\rho_{0}=0, n=30\right)$

\begin{tabular}{|c|c|c|c|c|c|c|c|c|c|c|c|}
\hline \multirow[b]{2}{*}{$\beta$} & \multirow[b]{2}{*}{$Y$} & \multirow[b]{2}{*}{ CP } & \multicolumn{3}{|c|}{$\alpha=0.10$} & \multicolumn{3}{|c|}{$\alpha=0.05$} & \multicolumn{3}{|c|}{$\alpha=0.01$} \\
\hline & & & $\mathrm{Zf}$ & $\mathrm{Zb}$ & Zc & $\mathrm{Zf}$ & $\mathrm{Zb}$ & Zc & $\mathrm{Zf}$ & $\mathrm{Zb}$ & Zc \\
\hline 1.2 & 4 & $z_{\alpha}$ & 0.1109 & 0.1270 & 0.0877 & 0.0651 & 0.0779 & 0.0372 & 0.0206 & 0.0423 & 0.0055 \\
\hline 3.0 & 14 & $t_{\alpha}$ & & 0.1213 & 0.0817 & & 0.0727 & 0.0323 & & 0.0406 & 0.0036 \\
\hline 1.2 & 4 & $z_{\alpha}$ & 0.1100 & 0.1375 & 0.0925 & 0.0623 & 0.0861 & 0.0393 & 0.0183 & 0.0488 & 0.0050 \\
\hline 3.0 & 25 & ta & & 0.1317 & 0.0862 & & 0.0803 & 0.0336 & & 0.0472 & 0.0034 \\
\hline 1.2 & 14 & $z_{\alpha}$ & 0.1081 & 0.1466 & 0.0936 & 0.0611 & 0.0974 & 0.0374 & 0.0213 & 0.0656 & 0.0054 \\
\hline 3.0 & 25 & $t_{\alpha}$ & & 0.1414 & 0.0875 & & 0.0922 & 0.0318 & & 0.0643 & 0.0037 \\
\hline-1.2 & 4 & $z a$ & 0.1127 & 0.1298 & 0.0899 & 0.0630 & 0.0760 & 0.0375 & 0.0206 & 0.0415 & 0.0053 \\
\hline-3.0 & 14 & $t_{\alpha}$ & & 0.1241 & 0.0837 & & 0.0711 & 0.0328 & & 0.0401 & 0.0034 \\
\hline-1.2 & 4 & $z_{\alpha}$ & 0.1108 & 0.1383 & 0.0926 & 0.0607 & 0.0844 & 0.0380 & 0.0188 & 0.0506 & 0.0054 \\
\hline-3.0 & 25 & $t_{\alpha}$ & & 0.1328 & 0.0869 & & 0.0789 & 0.0328 & & 0.0490 & 0.0034 \\
\hline-1.2 & 14 & $z a$ & 0.1041 & 0.1447 & 0.0916 & 0.0631 & 0.0974 & 0.0381 & 0.0204 & 0.0635 & 0.0051 \\
\hline-3.0 & 25 & ta & & 0.1391 & 0.0858 & & 0.0924 & 0.0326 & & 0.0625 & 0.0035 \\
\hline 3.0 & 25 & $z_{\alpha}$ & 0.1138 & 0.1403 & 0.0843 & 0.0695 & 0.0936 & 0.0336 & 0.0276 & 0.0630 & 0.0040 \\
\hline 3.0 & 25 & $t_{\alpha}$ & & 0.1354 & 0.0781 & & 0.0891 & 0.0287 & & 0.0616 & 0.0024 \\
\hline-3.0 & 25 & $z_{a}$ & 0.1113 & 0.1374 & 0.0817 & 0.0686 & 0.0923 & 0.0325 & 0.0276 & 0.0638 & 0.0042 \\
\hline-3.0 & 25 & $t_{\alpha}$ & & 0.1328 & 0.0761 & & 0.0875 & 0.0275 & & 0.0628 & 0.0028 \\
\hline
\end{tabular}


A NEW TEST FOR CORRELATION

1 Table 2. Comparison of type I error rates $(n=30, \alpha=0.05)$

\begin{tabular}{|c|c|c|c|c|c|c|c|c|c|c|c|c|c|c|}
\hline \multirow[b]{2}{*}{$\beta$} & \multirow[b]{2}{*}{$y$} & \multirow[b]{2}{*}{ CP } & \multicolumn{3}{|c|}{$\rho_{0}=0.50$} & \multicolumn{3}{|c|}{$\rho_{0}=0.60$} & \multicolumn{3}{|c|}{$\rho_{0}=0.75$} & \multicolumn{3}{|c|}{$\rho_{0}=0.90$} \\
\hline & & & $Z f$ & $\mathrm{Zb}$ & Zc & $\mathrm{Zf}$ & $\mathrm{Zb}$ & Zc & $Z f$ & $\mathrm{Zb}$ & Zc & $Z f$ & Zb & Zc \\
\hline 0.0 & 0 & $Z_{\alpha}$ & 0.0488 & 0.0335 & 0.0155 & 0.0497 & 0.0183 & 0.0053 & 0.0499 & 0.0081 & 0.0007 & 0.0496 & 0.0009 & 0.0000 \\
\hline 0.0 & 0 & $t_{\alpha}$ & & 0.0320 & 0.0130 & & 0.0189 & 0.0047 & & 0.0085 & 0.0006 & & 0.0009 & 0.0000 \\
\hline 0.0 & 1 & $z_{\alpha}$ & 0.0517 & 0.0537 & 0.0221 & 0.0525 & 0.0362 & 0.0092 & 0.0529 & 0.0218 & 0.0025 & 0.0543 & 0.0044 & 0.0002 \\
\hline 0.0 & 1 & $t_{\alpha}$ & & 0.0522 & 0.0186 & & 0.0373 & 0.0083 & & 0.0226 & 0.0023 & & 0.0044 & 0.0001 \\
\hline 0.0 & 10 & $z_{\alpha}$ & 0.0917 & 0.1477 & 0.0502 & 0.1025 & 0.1265 & 0.0302 & 0.1180 & 0.1037 & 0.0153 & 0.1346 & 0.0474 & 0.0045 \\
\hline 0.0 & 10 & $t_{\alpha}$ & & 0.1476 & 0.0441 & & 0.1293 & 0.0277 & & 0.1063 & 0.0143 & & 0.0478 & 0.0043 \\
\hline 0.5 & 0 & $z_{\alpha}$ & 0.0555 & 0.0344 & 0.0158 & 0.0550 & 0.0202 & 0.0058 & 0.0567 & 0.0106 & 0.0011 & 0.0590 & 0.0014 & 0.0001 \\
\hline 0.5 & 0 & $t_{\alpha}$ & & 0.0330 & 0.0133 & & 0.0205 & 0.0052 & & 0.0111 & 0.0010 & & 0.0014 & 0.0001 \\
\hline 1.0 & 0 & $z_{\alpha}$ & 0.0489 & 0.0269 & 0.0121 & 0.0449 & 0.0156 & 0.0037 & 0.0308 & 0.0128 & 0.0010 & 0.0078 & 0.0072 & 0.0000 \\
\hline 1.0 & 0 & $t_{\alpha}$ & & 0.0256 & 0.0096 & & 0.0162 & 0.0033 & & 0.0135 & 0.0009 & & 0.0073 & 0.0000 \\
\hline 0.5 & 1 & $z_{\alpha}$ & 0.0558 & 0.0548 & 0.0225 & 0.0558 & 0.0366 & 0.0087 & 0.0571 & 0.0230 & 0.0028 & 0.0580 & 0.0049 & 0.0002 \\
\hline 0.5 & 1 & $t_{\alpha}$ & & 0.0534 & 0.0194 & & 0.0375 & 0.0079 & & 0.0239 & 0.0026 & & 0.0050 & 0.0002 \\
\hline 1.2 & 4 & $z_{\alpha}$ & 0.0760 & 0.0998 & 0.0386 & 0.0809 & 0.0776 & 0.0198 & 0.0875 & 0.0597 & 0.0083 & 0.0931 & 0.0223 & 0.0016 \\
\hline 1.2 & 4 & $t_{\alpha}$ & & 0.0982 & 0.0337 & & 0.0790 & 0.0179 & & 0.0612 & 0.0076 & & 0.0224 & 0.0015 \\
\hline 1.2 & 10 & $z_{\alpha}$ & 0.0973 & 0.1505 & 0.0529 & 0.1053 & 0.1264 & 0.0309 & 0.1218 & 0.1036 & 0.0162 & 0.1370 & 0.0490 & 0.0044 \\
\hline 1.2 & 10 & $t_{\alpha}$ & & 0.1501 & 0.0470 & & 0.1288 & 0.0282 & & 0.1057 & 0.0153 & & 0.0494 & 0.0043 \\
\hline 1.2 & 25 & $z_{\alpha}$ & 0.1551 & 0.2064 & 0.0770 & 0.1735 & 0.1803 & 0.0533 & 0.2002 & 0.1566 & 0.0337 & 0.2268 & 0.0936 & 0.0137 \\
\hline 1.2 & 25 & $t_{\alpha}$ & & 0.2065 & 0.0697 & & 0.1829 & 0.0492 & & 0.1595 & 0.0321 & & 0.0943 & 0.0132 \\
\hline
\end{tabular}

4 Note: $\beta$ : skewness; $\gamma$ : kurtosis; CP: Critical Point 


\section{WANG \& SA}

1 Table 2 (continued). Comparison of type I error rates $(n=30, \alpha=0.05)$

\begin{tabular}{|c|c|c|c|c|c|c|c|c|c|c|c|c|c|c|}
\hline \multirow[b]{2}{*}{$\beta$} & \multirow[b]{2}{*}{$Y$} & \multirow[b]{2}{*}{ CP } & \multicolumn{3}{|c|}{$\rho_{0}=0.50$} & \multicolumn{3}{|c|}{$\rho_{0}=0.60$} & \multicolumn{3}{|c|}{$\rho_{0}=0.75$} & \multicolumn{3}{|c|}{$\rho_{0}=0.90$} \\
\hline & & & $\mathrm{Zf}$ & $\mathrm{Zb}$ & Zc & $\mathrm{Zf}$ & $\mathrm{Zb}$ & Zc & $\mathrm{Zf}$ & $\mathrm{Zb}$ & $\mathrm{Zc}$ & $\mathrm{Zf}$ & $\mathrm{Zb}$ & Zc \\
\hline-1.2 & 4 & $z_{\alpha}$ & 0.0762 & 0.0984 & 0.0376 & 0.0803 & 0.0777 & 0.0199 & 0.0864 & 0.0609 & 0.0087 & 0.0926 & 0.0228 & 0.0018 \\
\hline-1.2 & 4 & $t_{\alpha}$ & & 0.0968 & 0.0330 & & 0.0790 & 0.0182 & & 0.0623 & 0.0082 & & 0.0229 & 0.0017 \\
\hline-1.2 & 10 & $z_{\alpha}$ & 0.0955 & 0.1500 & 0.0515 & 0.1060 & 0.1265 & 0.0320 & 0.1224 & 0.1073 & 0.0170 & 0.1363 & 0.0485 & 0.0050 \\
\hline-1.2 & 10 & $t_{\alpha}$ & & 0.1501 & 0.0458 & & 0.1286 & 0.0290 & & 0.1097 & 0.0160 & & 0.0488 & 0.0049 \\
\hline-1.2 & 25 & $z_{\alpha}$ & 0.1580 & 0.2076 & 0.0791 & 0.1731 & 0.1791 & 0.0529 & 0.1994 & 0.1570 & 0.0339 & 0.2240 & 0.0939 & 0.0144 \\
\hline-1.2 & 25 & $t_{\alpha}$ & & 0.2068 & 0.0714 & & 0.1816 & 0.0490 & & 0.1601 & 0.0323 & & 0.0947 & 0.0138 \\
\hline 1.2 & 4 & $z_{\alpha}$ & 0.0837 & 0.1467 & 0.0511 & 0.0891 & 0.1318 & 0.0304 & 0.0864 & 0.0609 & 0.0087 & 0.1141 & 0.0664 & 0.0044 \\
\hline 1.2 & 10 & $t_{\alpha}$ & & 0.1470 & 0.0453 & & 0.1348 & 0.0277 & & 0.0623 & 0.0082 & & 0.0667 & 0.0043 \\
\hline 1.2 & 4 & $z_{\alpha}$ & 0.0996 & 0.2251 & 0.0668 & 0.1133 & 0.2153 & 0.0450 & 0.1224 & 0.1073 & 0.0170 & 0.2428 & 0.1272 & 0.0119 \\
\hline 1.2 & 25 & $t_{\alpha}$ & & 0.2269 & 0.0600 & & 0.2204 & 0.0418 & & 0.1097 & 0.0160 & & 0.1274 & 0.0113 \\
\hline-1.2 & 4 & $z_{\alpha}$ & 0.0836 & 0.1474 & 0.0510 & 0.0884 & 0.1302 & 0.0305 & 0.1994 & 0.1570 & 0.0339 & 0.1120 & 0.0682 & 0.0047 \\
\hline-1.2 & 10 & $t_{\alpha}$ & & 0.1472 & 0.0450 & & 0.1329 & 0.0276 & & 0.1601 & 0.0323 & & 0.0685 & 0.0044 \\
\hline-1.2 & 4 & $z_{\alpha}$ & 0.0993 & 0.2243 & 0.0685 & 0.1123 & 0.2181 & 0.0480 & 0.0998 & 0.1185 & 0.0150 & 0.2419 & 0.1270 & 0.0117 \\
\hline-1.2 & 25 & $t_{\alpha}$ & & 0.2263 & 0.0618 & & 0.2228 & 0.0443 & & 0.1213 & 0.0140 & & 0.1273 & 0.0111 \\
\hline 1.2 & 4 & $z_{\alpha}$ & 0.1037 & 0.1602 & 0.0628 & 0.1097 & 0.1332 & 0.0419 & 0.1360 & 0.2117 & 0.0283 & 0.1731 & 0.0189 & 0.0084 \\
\hline 3.0 & 14 & $t_{\alpha}$ & & 0.1585 & 0.0560 & & 0.1342 & 0.0384 & & 0.2149 & 0.0267 & & 0.0191 & 0.0082 \\
\hline 1.2 & 4 & $z_{\alpha}$ & 0.1134 & 0.2081 & 0.0737 & 0.1220 & 0.1919 & 0.0512 & 0.0993 & 0.1181 & 0.0154 & 0.1694 & 0.0871 & 0.0163 \\
\hline 3.0 & 25 & $t_{\alpha}$ & & 0.2085 & 0.0669 & & 0.1946 & 0.0475 & & 0.1210 & 0.0144 & & 0.0872 & 0.0155 \\
\hline
\end{tabular}

$4 \quad$ Note: $\beta$ : skewness; $\gamma$ : kurtosis; CP: Critical Point 


\section{A NEW TEST FOR CORRELATION}

1 Table 2 (continued). Comparison of type I error rates $(n=30, \alpha=0.05)$

\begin{tabular}{|c|c|c|c|c|c|c|c|c|c|c|c|c|c|c|}
\hline \multirow[b]{2}{*}{$\beta$} & \multirow[b]{2}{*}{$y$} & \multirow[b]{2}{*}{$\mathrm{CP}$} & \multicolumn{3}{|c|}{$\rho_{0}=0.50$} & \multicolumn{3}{|c|}{$\rho_{0}=0.60$} & \multicolumn{3}{|c|}{$\rho_{0}=0.75$} & \multicolumn{3}{|c|}{$\rho_{0}=0.90$} \\
\hline & & & $\mathrm{Zf}$ & $\mathrm{Zb}$ & $\mathrm{Zc}$ & $\mathrm{Zf}$ & $\mathrm{Zb}$ & $\mathrm{Zc}$ & Zf & $\mathrm{Zb}$ & $\mathrm{Zc}$ & $Z f$ & $\mathrm{Zb}$ & Zc \\
\hline 1.2 & 14 & $z_{\alpha}$ & 0.1364 & 0.1994 & 0.0757 & 0.1533 & 0.1719 & 0.0518 & 0.1363 & 0.2096 & 0.0293 & 0.1960 & 0.0680 & 0.0142 \\
\hline 3.0 & 25 & $t_{\alpha}$ & & 0.1983 & 0.0683 & & 0.1739 & 0.0480 & & 0.2133 & 0.0276 & & 0.0683 & 0.0137 \\
\hline-1.2 & 4 & $z_{\alpha}$ & 0.1016 & 0.1565 & 0.0623 & 0.1089 & 0.1298 & 0.0405 & 0.1151 & 0.0949 & 0.0212 & 0.1734 & 0.0194 & 0.0083 \\
\hline-3.0 & 14 & $t_{\alpha}$ & & 0.1550 & 0.0558 & & 0.1304 & 0.0369 & & 0.0964 & 0.0199 & & 0.0196 & 0.0082 \\
\hline-1.2 & 4 & $z_{\alpha}$ & 0.1115 & 0.2082 & 0.0723 & 0.1239 & 0.1912 & 0.0521 & 0.1419 & 0.1719 & 0.0344 & 0.1682 & 0.0845 & 0.0155 \\
\hline-3.0 & 25 & $t_{\alpha}$ & & 0.2082 & 0.0652 & & 0.1942 & 0.0481 & & 0.1742 & 0.0325 & & 0.0847 & 0.0148 \\
\hline-1.2 & 14 & $z_{\alpha}$ & 0.1365 & 0.1981 & 0.0733 & 0.1534 & 0.1733 & 0.0526 & 0.1759 & 0.1429 & 0.0344 & 0.1931 & 0.0673 & 0.0141 \\
\hline-3.0 & 25 & $t_{\alpha}$ & & 0.1976 & 0.0659 & & 0.1747 & 0.0484 & & 0.1453 & 0.0327 & & 0.0676 & 0.0136 \\
\hline 3.0 & 25 & $z_{\alpha}$ & 0.1648 & 0.2193 & 0.0833 & 0.1852 & 0.1898 & 0.0588 & 0.1147 & 0.0943 & 0.0216 & 0.2290 & 0.1044 & 0.0156 \\
\hline 3.0 & 25 & $t_{\alpha}$ & & 0.2183 & 0.0755 & & 0.1913 & 0.0547 & & 0.0958 & 0.0205 & & 0.1049 & 0.0150 \\
\hline-3.0 & 25 & $z_{\alpha}$ & 0.1654 & 0.2186 & 0.0833 & 0.1820 & 0.1922 & 0.0587 & 0.1402 & 0.1696 & 0.0326 & 0.2257 & 0.1068 & 0.0159 \\
\hline-3.0 & 25 & $t_{\alpha}$ & & 0.2172 & 0.0754 & & 0.1938 & 0.0543 & & 0.1723 & 0.0308 & & 0.1074 & 0.0152 \\
\hline
\end{tabular}

4 Note: $\beta$ : skewness; $\gamma$ : kurtosis; CP: Critical Point 


\section{WANG \& SA}

1 Table 3. Comparison of type I error rates $(n=30, \alpha=0.01)$

\begin{tabular}{|c|c|c|c|c|c|c|c|c|c|c|c|c|c|c|}
\hline \multirow[b]{2}{*}{$\beta$} & \multirow[b]{2}{*}{$y$} & \multirow[b]{2}{*}{ CP } & \multicolumn{3}{|c|}{$\rho_{0}=0.50$} & \multicolumn{3}{|c|}{$\rho_{0}=0.60$} & \multicolumn{3}{|c|}{$\rho_{0}=0.75$} & \multicolumn{3}{|c|}{$\rho_{0}=0.90$} \\
\hline & & & $Z f$ & $\mathrm{Zb}$ & Zc & $\mathrm{Zf}$ & $\mathrm{Zb}$ & Zc & $Z f$ & $\mathrm{Zb}$ & Zc & $Z f$ & Zb & Zc \\
\hline 0.0 & 0 & $Z_{\alpha}$ & 0.0107 & 0.0352 & 0.0034 & 0.0107 & 0.0272 & 0.0017 & 0.0103 & 0.0137 & 0.0003 & 0.0103 & 0.0013 & 0.0000 \\
\hline 0.0 & 0 & $t_{\alpha}$ & & 0.0375 & 0.0027 & & 0.0297 & 0.0014 & & 0.0145 & 0.0002 & & 0.0013 & 0.0000 \\
\hline 0.0 & 1 & $z_{\alpha}$ & 0.0115 & 0.0627 & 0.0056 & 0.0118 & 0.0526 & 0.0034 & 0.0118 & 0.0315 & 0.0008 & 0.0123 & 0.0049 & 0.0001 \\
\hline 0.0 & 1 & $t_{\alpha}$ & & 0.0671 & 0.0045 & & 0.0562 & 0.0027 & & 0.0330 & 0.0007 & & 0.0050 & 0.0001 \\
\hline 0.0 & 10 & $z_{\alpha}$ & 0.0350 & 0.1768 & 0.0160 & 0.0417 & 0.1615 & 0.0125 & 0.0512 & 0.1263 & 0.0072 & 0.0611 & 0.0501 & 0.0023 \\
\hline 0.0 & 10 & $t_{\alpha}$ & & 0.1858 & 0.0136 & & 0.1694 & 0.0108 & & 0.1295 & 0.0062 & & 0.0502 & 0.0019 \\
\hline 0.5 & 0 & $z_{\alpha}$ & 0.0125 & 0.0359 & 0.0036 & 0.0126 & 0.0285 & 0.0017 & 0.0135 & 0.0158 & 0.0004 & 0.0138 & 0.0017 & 0.0000 \\
\hline 0.5 & 0 & $t_{\alpha}$ & & 0.0386 & 0.0030 & & 0.0308 & 0.0014 & & 0.0167 & 0.0004 & & 0.0017 & 0.0000 \\
\hline 1.0 & 0 & $z_{\alpha}$ & 0.0138 & 0.0274 & 0.0025 & 0.0124 & 0.0245 & 0.0012 & 0.0083 & 0.0201 & 0.0003 & 0.0013 & 0.0093 & 0.0000 \\
\hline 1.0 & 0 & $t_{\alpha}$ & & 0.0293 & 0.0020 & & 0.0265 & 0.0010 & & 0.0215 & 0.0002 & & 0.0097 & 0.0000 \\
\hline 0.5 & 1 & $z_{\alpha}$ & 0.0124 & 0.0612 & 0.0054 & 0.0132 & 0.0513 & 0.0032 & 0.0138 & 0.0310 & 0.0009 & 0.0145 & 0.0057 & 0.0001 \\
\hline 0.5 & 1 & $t_{\alpha}$ & & 0.0651 & 0.0044 & & 0.0543 & 0.0026 & & 0.0325 & 0.0008 & & 0.0057 & 0.0001 \\
\hline 1.2 & 4 & $z_{\alpha}$ & 0.0246 & 0.1114 & 0.0114 & 0.0258 & 0.1011 & 0.0081 & 0.0296 & 0.0753 & 0.0042 & 0.0338 & 0.0236 & 0.0009 \\
\hline 1.2 & 4 & $t_{\alpha}$ & & 0.1175 & 0.0095 & & 0.1060 & 0.0067 & & 0.0778 & 0.0037 & & 0.0236 & 0.0008 \\
\hline 1.2 & 10 & $z_{\alpha}$ & 0.0378 & 0.1754 & 0.0173 & 0.0432 & 0.1597 & 0.0126 & 0.0538 & 0.1259 & 0.0083 & 0.0643 & 0.0528 & 0.0028 \\
\hline 1.2 & 10 & $t_{\alpha}$ & & 0.1834 & 0.0144 & & 0.1662 & 0.0108 & & 0.1292 & 0.0073 & & 0.0530 & 0.0025 \\
\hline 1.2 & 25 & $z_{\alpha}$ & 0.0833 & 0.2346 & 0.0292 & 0.0989 & 0.2168 & 0.0260 & 0.1169 & 0.1826 & 0.0187 & 0.1383 & 0.1010 & 0.0099 \\
\hline 1.2 & 25 & $t_{\alpha}$ & & 0.2430 & 0.0251 & & 0.2238 & 0.0228 & & 0.1866 & 0.0169 & & 0.1014 & 0.0091 \\
\hline
\end{tabular}

$4 \quad$ Note: $\beta$ : skewness; $\gamma$ : kurtosis; CP: Critical Point 


\section{A NEW TEST FOR CORRELATION}

1 Table 3 (continued). Comparison of type I error rates $(n=30, \alpha=0.01)$

\begin{tabular}{|c|c|c|c|c|c|c|c|c|c|c|c|c|c|c|}
\hline \multirow[b]{2}{*}{$\beta$} & \multirow[b]{2}{*}{$y$} & \multirow[b]{2}{*}{ CP } & \multicolumn{3}{|c|}{$\rho_{0}=0.50$} & \multicolumn{3}{|c|}{$\rho_{0}=0.60$} & \multicolumn{3}{|c|}{$\rho_{0}=0.75$} & \multicolumn{3}{|c|}{$\rho_{0}=0.90$} \\
\hline & & & $Z f$ & $\mathrm{Zb}$ & Zc & $\mathrm{Zf}$ & $\mathrm{Zb}$ & Zc & $Z f$ & $\mathrm{Zb}$ & Zc & $Z f$ & Zb & Zc \\
\hline-1.2 & 4 & $Z_{\alpha}$ & 0.0237 & 0.1116 & 0.0110 & 0.0264 & 0.1010 & 0.0075 & 0.0300 & 0.0749 & 0.0039 & 0.0336 & 0.0243 & 0.0008 \\
\hline-1.2 & 4 & $t_{\alpha}$ & & 0.1173 & 0.0092 & & 0.1064 & 0.0064 & & 0.0774 & 0.0034 & & 0.0244 & 0.0006 \\
\hline-1.2 & 10 & $z_{\alpha}$ & 0.0381 & 0.1780 & 0.0175 & 0.0439 & 0.1605 & 0.0131 & 0.0531 & 0.1241 & 0.0080 & 0.0628 & 0.0519 & 0.0028 \\
\hline-1.2 & 10 & $t_{\alpha}$ & & 0.1860 & 0.0146 & & 0.1676 & 0.0113 & & 0.1270 & 0.0071 & & 0.0521 & 0.0025 \\
\hline-1.2 & 25 & $z_{\alpha}$ & 0.0833 & 0.2349 & 0.0295 & 0.0977 & 0.2169 & 0.0258 & 0.1206 & 0.1837 & 0.0196 & 0.1363 & 0.0997 & 0.0091 \\
\hline-1.2 & 25 & $t_{\alpha}$ & & 0.2437 & 0.0254 & & 0.2240 & 0.0226 & & 0.1878 & 0.0178 & & 0.1002 & 0.0085 \\
\hline 1.2 & 4 & $z_{\alpha}$ & 0.0286 & 0.1740 & 0.0165 & 0.0318 & 0.1676 & 0.0120 & 0.0364 & 0.1403 & 0.0069 & 0.0446 & 0.0689 & 0.0023 \\
\hline 1.2 & 10 & $t_{\alpha}$ & & 0.1819 & 0.0136 & & 0.1752 & 0.0101 & & 0.1434 & 0.0061 & & 0.0692 & 0.0020 \\
\hline 1.2 & 4 & $z_{\alpha}$ & 0.0400 & 0.2715 & 0.0247 & 0.0457 & 0.2683 & 0.0201 & 0.0567 & 0.2376 & 0.0145 & 0.0995 & 0.1277 & 0.0064 \\
\hline 1.2 & 25 & $t_{\alpha}$ & & 0.2829 & 0.0206 & & 0.2774 & 0.0174 & & 0.2407 & 0.0126 & & 0.1278 & 0.0057 \\
\hline-1.2 & 4 & $z_{\alpha}$ & 0.0281 & 0.1730 & 0.0161 & 0.0319 & 0.1672 & 0.0125 & 0.0371 & 0.1417 & 0.0072 & 0.0446 & 0.0698 & 0.0023 \\
\hline-1.2 & 10 & $t_{\alpha}$ & & 0.1813 & 0.0133 & & 0.1753 & 0.0105 & & 0.1448 & 0.0060 & & 0.0699 & 0.0020 \\
\hline-1.2 & 4 & $z_{\alpha}$ & 0.0390 & 0.2699 & 0.0244 & 0.0452 & 0.2660 & 0.0199 & 0.0575 & 0.2371 & 0.0140 & 0.1003 & 0.1284 & 0.0067 \\
\hline-1.2 & 25 & $t_{\alpha}$ & & 0.2813 & 0.0203 & & 0.2752 & 0.0172 & & 0.2400 & 0.0123 & & 0.1285 & 0.0059 \\
\hline 1.2 & 4 & $z_{\alpha}$ & 0.0414 & 0.1707 & 0.0213 & 0.0436 & 0.1560 & 0.0166 & 0.0481 & 0.1080 & 0.0108 & 0.0818 & 0.0208 & 0.0059 \\
\hline 3.0 & 14 & $t_{\alpha}$ & & 0.1777 & 0.0177 & & 0.1623 & 0.0142 & & 0.1101 & 0.0094 & & 0.0211 & 0.0054 \\
\hline 1.2 & 4 & $z_{\alpha}$ & 0.0468 & 0.2369 & 0.0275 & 0.0512 & 0.2279 & 0.0234 & 0.0605 & 0.1915 & 0.0182 & 0.0621 & 0.0865 & 0.0089 \\
\hline 3.0 & 25 & $t_{\alpha}$ & & 0.2463 & 0.0231 & & 0.2353 & 0.0204 & & 0.1946 & 0.0161 & & 0.0866 & 0.0078 \\
\hline
\end{tabular}

$4 \quad$ Note: $\beta$ : skewness; $\gamma$ : kurtosis; $C P$ : Critical Point 


\section{WANG \& SA}

1 Table 3 (continued). Comparison of type I error rates $(n=30, \alpha=0.01)$

\begin{tabular}{|c|c|c|c|c|c|c|c|c|c|c|c|c|c|c|}
\hline \multirow[b]{2}{*}{$\beta$} & \multirow[b]{2}{*}{$Y$} & \multirow[b]{2}{*}{$\mathrm{CP}$} & \multicolumn{3}{|c|}{$\rho_{0}=0.50$} & \multicolumn{3}{|c|}{$\rho_{0}=0.60$} & \multicolumn{3}{|c|}{$\rho_{0}=0.75$} & \multicolumn{3}{|c|}{$\rho_{0}=0.90$} \\
\hline & & & $Z f$ & $\mathrm{Zb}$ & Zc & $Z f$ & $\mathrm{Zb}$ & Zc & $Z f$ & $\mathrm{Zb}$ & Zc & $Z f$ & $\mathrm{Zb}$ & Zc \\
\hline 1.2 & 14 & $z_{\alpha}$ & 0.0682 & 0.2232 & 0.0290 & 0.0783 & 0.2079 & 0.0248 & 0.0940 & 0.1625 & 0.0187 & 0.1061 & 0.0709 & 0.0090 \\
\hline 3.0 & 25 & $t_{\alpha}$ & & 0.2311 & 0.0252 & & 0.2145 & 0.0219 & & 0.1657 & 0.0165 & & 0.0712 & 0.0081 \\
\hline-1.2 & 4 & $z_{\alpha}$ & 0.0425 & 0.1756 & 0.0223 & 0.0449 & 0.1566 & 0.0171 & 0.0479 & 0.1088 & 0.0107 & 0.0809 & 0.0205 & 0.0062 \\
\hline-3.0 & 14 & $t_{\alpha}$ & & 0.1826 & 0.0192 & & 0.1626 & 0.0150 & & 0.1108 & 0.0092 & & 0.0207 & 0.0059 \\
\hline-1.2 & 4 & $z_{\alpha}$ & 0.0466 & 0.2365 & 0.0269 & 0.0531 & 0.2306 & 0.0238 & 0.0593 & 0.1913 & 0.0171 & 0.0607 & 0.0880 & 0.0084 \\
\hline-3.0 & 25 & $t_{\alpha}$ & & 0.2454 & 0.0225 & & 0.2375 & 0.0209 & & 0.1943 & 0.0150 & & 0.0882 & 0.0075 \\
\hline-1.2 & 14 & $z_{\alpha}$ & 0.0671 & 0.2213 & 0.0283 & 0.0779 & 0.2024 & 0.0248 & 0.0939 & 0.1612 & 0.0179 & 0.1071 & 0.0708 & 0.0093 \\
\hline-3.0 & 25 & $t_{\alpha}$ & & 0.2291 & 0.0242 & & 0.2098 & 0.0218 & & 0.1642 & 0.0163 & & 0.0711 & 0.0085 \\
\hline 3.0 & 25 & $z_{\alpha}$ & 0.0934 & 0.2429 & 0.0345 & 0.1058 & 0.2221 & 0.0295 & 0.1249 & 0.1873 & 0.0217 & 0.1403 & 0.1080 & 0.0098 \\
\hline 3.0 & 25 & $t_{\alpha}$ & & 0.2501 & 0.0300 & & 0.2285 & 0.0262 & & 0.1912 & 0.0197 & & 0.1085 & 0.0088 \\
\hline-3.0 & 25 & $z_{\alpha}$ & 0.0936 & 0.2375 & 0.0345 & 0.1060 & 0.2196 & 0.0290 & 0.1241 & 0.1872 & 0.0206 & 0.1406 & 0.1094 & 0.0098 \\
\hline-3.0 & 25 & $t_{\alpha}$ & & 0.2446 & 0.0301 & & 0.2262 & 0.0253 & & 0.1907 & 0.0188 & & 0.1100 & 0.0090 \\
\hline
\end{tabular}

4 Note: $\beta$ : skewness; $\gamma$ : kurtosis; CP: Critical Point 


\section{A NEW TEST FOR CORRELATION}

1 Table 4. Power performance for test $\rho_{0}=0(n=30, \alpha=0.05)$

\begin{tabular}{|c|c|c|c|c|c|c|c|c|c|c|c|c|c|c|c|c|}
\hline \multirow[b]{2}{*}{ b } & \multirow[b]{2}{*}{ g } & \multicolumn{3}{|c|}{$\mathrm{ra}=0.0$} & \multicolumn{3}{|c|}{$\mathrm{ra}=0.2$} & \multicolumn{3}{|c|}{$\mathrm{ra}=0.4$} & \multicolumn{3}{|c|}{$\mathrm{ra}=0.6$} & \multicolumn{3}{|c|}{$\mathrm{ra}=0.8$} \\
\hline & & $\mathrm{Zf}$ & $\mathrm{Zb}$ & $\mathrm{Zc}$ & $\mathrm{Zf}$ & $\mathrm{Zb}$ & $\mathrm{Zc}$ & $\mathrm{Zf}$ & $\mathrm{Zb}$ & $\mathrm{Zc}$ & $\mathrm{Zf}$ & $\mathrm{Zb}$ & $\mathrm{Zc}$ & $\mathrm{Zf}$ & $\mathrm{Zb}$ & $\mathrm{Zc}$ \\
\hline 0.0 & 0 & 0.0498 & 0.0683 & 0.0635 & 0.2778 & 0.3148 & 0.3002 & 0.7231 & 0.7376 & 0.7156 & 0.9775 & 0.9785 & 0.9727 & 1.0000 & 1.0000 & 0.9993 \\
\hline 0.0 & 0 & & 0.0624 & 0.0576 & & 0.2976 & 0.2827 & & 0.7192 & 0.6950 & & 0.9749 & 0.9674 & & 1.0000 & 0.9991 \\
\hline 0.0 & 1 & 0.0494 & 0.0739 & 0.0626 & 0.2814 & 0.3321 & 0.2964 & 0.7227 & 0.7489 & 0.6980 & 0.9776 & 0.9798 & 0.9546 & 1.0000 & 1.0000 & 0.9840 \\
\hline 0.0 & 1 & & 0.0681 & 0.0565 & & 0.3133 & 0.2778 & & 0.7314 & 0.6758 & & 0.9768 & 0.9473 & & 0.9999 & 0.9815 \\
\hline 0.0 & 10 & 0.0553 & 0.0991 & 0.0485 & 0.3030 & 0.3849 & 0.2641 & 0.7436 & 0.7942 & 0.6416 & 0.9809 & 0.9837 & 0.8472 & 1.0000 & 0.9998 & 0.8527 \\
\hline 0.0 & 10 & & 0.0933 & 0.0427 & & 0.3653 & 0.2419 & & 0.7772 & 0.6128 & & 0.9811 & 0.8330 & & 0.9997 & 0.8428 \\
\hline 0.5 & 0 & 0.0502 & 0.0625 & 0.0585 & 0.2821 & 0.3074 & 0.2927 & 0.7201 & 0.7334 & 0.7089 & 0.9766 & 0.9771 & 0.9680 & 1.0000 & 0.9999 & 0.9977 \\
\hline 0.5 & 0 & & 0.0564 & 0.0528 & & 0.2888 & 0.2738 & & 0.7147 & 0.6882 & & 0.9738 & 0.9622 & & 0.9998 & 0.9969 \\
\hline 1.0 & 0 & 0.0553 & 0.0550 & 0.0511 & 0.2655 & 0.2531 & 0.2377 & 0.6519 & 0.6278 & 0.5973 & 0.9409 & 0.9341 & 0.9147 & 0.9988 & 0.9981 & 0.9957 \\
\hline 1.0 & 0 & & 0.0493 & 0.0457 & & 0.2350 & 0.2193 & & 0.6049 & 0.5726 & & 0.9254 & 0.9022 & & 0.9978 & 0.9948 \\
\hline 0.5 & 1 & 0.0514 & 0.0704 & 0.0602 & 0.2832 & 0.3254 & 0.2907 & 0.7209 & 0.7451 & 0.6952 & 0.9763 & 0.9783 & 0.9497 & 1.0000 & 0.9999 & 0.9808 \\
\hline 0.5 & 1 & & 0.0650 & 0.0548 & & 0.3063 & 0.2719 & & 0.7263 & 0.6721 & & 0.9753 & 0.9419 & & 0.9999 & 0.9783 \\
\hline 1.2 & 4 & 0.0570 & 0.0744 & 0.0510 & 0.2927 & 0.3284 & 0.2571 & 0.7218 & 0.7473 & 0.6451 & 0.9755 & 0.9765 & 0.8935 & 1.0000 & 0.9994 & 0.9213 \\
\hline 1.2 & 4 & & 0.0686 & 0.0452 & & 0.3090 & 0.2368 & & 0.7286 & 0.6192 & & 0.9728 & 0.8809 & & 0.9994 & 0.9146 \\
\hline 1.2 & 10 & 0.0565 & 0.0919 & 0.0467 & 0.3046 & 0.3716 & 0.2568 & 0.7415 & 0.7841 & 0.6343 & 0.9798 & 0.9808 & 0.8440 & 1.0000 & 0.9997 & 0.8543 \\
\hline 1.2 & 10 & & 0.0863 & 0.0408 & & 0.3518 & 0.2342 & & 0.7672 & 0.6059 & & 0.9780 & 0.8295 & & 0.9996 & 0.8442 \\
\hline 1.2 & 25 & 0.0597 & 0.1033 & 0.0359 & 0.3213 & 0.4029 & 0.2392 & 0.7433 & 0.7969 & 0.5898 & 0.9736 & 0.9780 & 0.7636 & 0.9999 & 0.9997 & 0.7636 \\
\hline 1.2 & 25 & & 0.0988 & 0.0311 & & 0.3831 & 0.2147 & & 0.7802 & 0.5592 & & 0.9743 & 0.7470 & & 0.9996 & 0.7519 \\
\hline-1.2 & 4 & 0.0556 & 0.0725 & 0.0493 & 0.2916 & 0.3273 & 0.2562 & 0.7208 & 0.7472 & 0.6445 & 0.9755 & 0.9764 & 0.8931 & 1.0000 & 0.9995 & 0.9211 \\
\hline-1.2 & 4 & & 0.0666 & 0.0436 & & 0.3078 & 0.2363 & & 0.7282 & 0.6183 & & 0.9729 & 0.8809 & & 0.9994 & 0.9141 \\
\hline
\end{tabular}

4 Note: $\beta$ : skewness; $\gamma$ : kurtosis; the "Zf", "Zb", and "Zc" results are calculated using the critical points $z_{\alpha}$ and $t_{\alpha}$ as the first and the second number 


\section{WANG \& SA}

1 Table 4 (continued). Power performance for test $\rho_{0}=0(n=30, \alpha=0.05)$

\begin{tabular}{|c|c|c|c|c|c|c|c|c|c|c|c|c|c|c|c|c|}
\hline \multirow[b]{2}{*}{ b } & \multirow[b]{2}{*}{ g } & \multicolumn{3}{|c|}{$\mathrm{ra}=0.0$} & \multicolumn{3}{|c|}{$\mathrm{ra}=0.2$} & \multicolumn{3}{|c|}{$\mathrm{ra}=0.4$} & \multicolumn{3}{|c|}{$\mathrm{ra}=0.6$} & \multicolumn{3}{|c|}{$\mathrm{ra}=0.8$} \\
\hline & & $\mathrm{Zf}$ & $\mathrm{Zb}$ & $\mathrm{Zc}$ & $\mathrm{Zf}$ & $\mathrm{Zb}$ & $\mathrm{Zc}$ & $\mathrm{Zf}$ & $\mathrm{Zb}$ & $\mathrm{Zc}$ & Zf & $\mathrm{Zb}$ & $\mathrm{Zc}$ & $\mathrm{Zf}$ & $\mathrm{Zb}$ & Zc \\
\hline-1.2 & 10 & 0.0578 & 0.0925 & 0.0457 & 0.3050 & 0.3731 & 0.2574 & 0.7384 & 0.7819 & 0.6315 & 0.9790 & 0.9803 & 0.8443 & 1.0000 & 0.9995 & 0.8541 \\
\hline-1.2 & 10 & & 0.0868 & 0.0399 & & 0.3534 & 0.2344 & & 0.7640 & 0.6029 & & 0.9772 & 0.8305 & & 0.9994 & 0.8444 \\
\hline-1.2 & 25 & 0.0597 & 0.1034 & 0.0362 & 0.3217 & 0.4027 & 0.2388 & 0.7440 & 0.7974 & 0.5898 & 0.9731 & 0.9775 & 0.7606 & 0.9999 & 0.9997 & 0.7667 \\
\hline-1.2 & 25 & & 0.0986 & 0.0307 & & 0.3836 & 0.2139 & & 0.7801 & 0.5588 & & 0.9739 & 0.7430 & & 0.9996 & 0.7542 \\
\hline 1.2 & 4 & 0.0565 & 0.0827 & 0.0472 & 0.3013 & 0.3557 & 0.2615 & 0.7359 & 0.7704 & 0.6452 & 0.9799 & 0.9802 & 0.8731 & 1.0000 & 0.9996 & 0.8910 \\
\hline 1.2 & 10 & & 0.0768 & 0.0413 & & 0.3353 & 0.2396 & & 0.7525 & 0.6178 & & 0.9774 & 0.8600 & & 0.9995 & 0.8821 \\
\hline 1.2 & 4 & 0.0577 & 0.0956 & 0.0438 & 0.3152 & 0.3844 & 0.2569 & 0.7635 & 0.8037 & 0.6410 & 0.9864 & 0.9867 & 0.8343 & 1.0000 & 0.9999 & 0.8378 \\
\hline 1.2 & 25 & & 0.0897 & 0.0378 & & 0.3647 & 0.2332 & & 0.7874 & 0.6125 & & 0.9847 & 0.8206 & & 0.9999 & 0.8267 \\
\hline-1.2 & 4 & 0.0564 & 0.0843 & 0.0489 & 0.2957 & 0.3508 & 0.2590 & 0.7336 & 0.7677 & 0.6426 & 0.9802 & 0.9812 & 0.8737 & 1.0000 & 0.9997 & 0.8895 \\
\hline-1.2 & 10 & & 0.0783 & 0.0433 & & 0.3313 & 0.2374 & & 0.7494 & 0.6149 & & 0.9783 & 0.8608 & & 0.9996 & 0.8811 \\
\hline-1.2 & 4 & 0.0571 & 0.0945 & 0.0431 & 0.3139 & 0.3849 & 0.2559 & 0.7627 & 0.8034 & 0.6414 & 0.9858 & 0.9868 & 0.8364 & 1.0000 & 0.9999 & 0.8388 \\
\hline-1.2 & 25 & & 0.0889 & 0.0370 & & 0.3656 & 0.2336 & & 0.7867 & 0.6125 & & 0.9846 & 0.8227 & & 0.9999 & 0.8289 \\
\hline 1.2 & 4 & 0.0633 & 0.0766 & 0.0372 & 0.3067 & 0.3263 & 0.2117 & 0.7135 & 0.7313 & 0.5620 & 0.9708 & 0.9731 & 0.8130 & 0.9999 & 0.9996 & 0.8384 \\
\hline 3.0 & 14 & & 0.0708 & 0.0323 & & 0.3084 & 0.1918 & & 0.7113 & 0.5319 & & 0.9688 & 0.7957 & & 0.9995 & 0.8275 \\
\hline 1.2 & 4 & 0.0618 & 0.0849 & 0.0384 & 0.3182 & 0.3593 & 0.2356 & 0.7479 & 0.7744 & 0.6102 & 0.9804 & 0.9804 & 0.8228 & 1.0000 & 0.9997 & 0.8327 \\
\hline 3.0 & 25 & & 0.0792 & 0.0331 & & 0.3397 & 0.2134 & & 0.7557 & 0.5806 & & 0.9775 & 0.8087 & & 0.9996 & 0.8220 \\
\hline 1.2 & 14 & 0.0629 & 0.0976 & 0.0383 & 0.3200 & 0.3859 & 0.2394 & 0.7464 & 0.7894 & 0.6004 & 0.9769 & 0.9785 & 0.7878 & 1.0000 & 0.9996 & 0.7894 \\
\hline 3.0 & 25 & & 0.0928 & 0.0330 & & 0.3665 & 0.2153 & & 0.7716 & 0.5698 & & 0.9749 & 0.7715 & & 0.9995 & 0.7781 \\
\hline-1.2 & 4 & 0.0638 & 0.0766 & 0.0369 & 0.3093 & 0.3297 & 0.2151 & 0.7127 & 0.7317 & 0.5633 & 0.9714 & 0.9738 & 0.8133 & 0.9999 & 0.9995 & 0.8377 \\
\hline-3.0 & 14 & & 0.0715 & 0.0317 & & 0.3107 & 0.1946 & & 0.7123 & 0.5345 & & 0.9698 & 0.7967 & & 0.9993 & 0.8266 \\
\hline
\end{tabular}

4 Note: $\beta$ : skewness; $\gamma$ : kurtosis; the "Zf", "Zb", and "Zc" results are calculated using the critical points $z_{\alpha}$ and $t_{\alpha}$ as the first and the second number 


\section{A NEW TEST FOR CORRELATION}

2 Table 4 (continued). Power performance for test $\rho_{0}=0(n=30, \alpha=0.05)$

\begin{tabular}{|c|c|c|c|c|c|c|c|c|c|c|c|c|c|c|c|c|}
\hline \multirow[b]{2}{*}{ b } & \multirow[b]{2}{*}{ g } & \multicolumn{3}{|c|}{$\mathrm{ra}=0.0$} & \multicolumn{3}{|c|}{$\mathrm{ra}=0.2$} & \multicolumn{3}{|c|}{$\mathrm{ra}=0.4$} & \multicolumn{3}{|c|}{$\mathrm{ra}=0.6$} & \multicolumn{3}{|c|}{$\mathrm{ra}=0.8$} \\
\hline & & $\mathrm{Zf}$ & $\mathrm{Zb}$ & $\mathrm{Zc}$ & $\mathrm{Zf}$ & $\mathrm{Zb}$ & $\mathrm{Zc}$ & $\mathrm{Zf}$ & $\mathrm{Zb}$ & $\mathrm{Zc}$ & Zf & $\mathrm{Zb}$ & $\mathrm{Zc}$ & $\mathrm{Zf}$ & $\mathrm{Zb}$ & Zc \\
\hline-1.2 & 4 & 0.0622 & 0.0855 & 0.0388 & 0.3186 & 0.3602 & 0.2388 & 0.7484 & 0.7755 & 0.6131 & 0.9806 & 0.9803 & 0.8225 & 1.0000 & 0.9997 & 0.8325 \\
\hline-3.0 & 25 & & 0.0802 & 0.0334 & & 0.3403 & 0.2159 & & 0.7568 & 0.5834 & & 0.9772 & 0.8074 & & 0.9996 & 0.8216 \\
\hline-1.2 & 14 & 0.0618 & 0.0967 & 0.0378 & 0.3200 & 0.3855 & 0.2395 & 0.7459 & 0.7878 & 0.5980 & 0.9776 & 0.9785 & 0.7889 & 1.0000 & 0.9996 & 0.7923 \\
\hline-3.0 & 25 & & 0.0918 & 0.0323 & & 0.3662 & 0.2161 & & 0.7702 & 0.5681 & & 0.9752 & 0.7738 & & 0.9996 & 0.7813 \\
\hline 3.0 & 25 & 0.0689 & 0.0935 & 0.0331 & 0.3289 & 0.3661 & 0.2129 & 0.7241 & 0.7498 & 0.5456 & 0.9625 & 0.9629 & 0.7458 & 0.9999 & 0.9991 & 0.7631 \\
\hline 3.0 & 25 & & 0.0889 & 0.0279 & & 0.3489 & 0.1918 & & 0.7311 & 0.5149 & & 0.9574 & 0.7274 & & 0.9990 & 0.7509 \\
\hline-3.0 & 25 & 0.0682 & 0.0919 & 0.0334 & 0.3293 & 0.3686 & 0.2146 & 0.7257 & 0.7508 & 0.5431 & 0.9625 & 0.9629 & 0.7451 & 0.9998 & 0.9992 & 0.7676 \\
\hline-3.0 & 25 & & 0.0875 & 0.0286 & & 0.3502 & 0.1926 & & 0.7317 & 0.5121 & & 0.9573 & 0.7276 & & 0.9991 & 0.7556 \\
\hline
\end{tabular}

$5 \quad$ Note: $\beta$ : skewness; $\gamma$ : kurtosis; the "Zf", "Zb", and "Zc" results are calculated using the critical points $z_{\alpha}$ and $t_{\alpha}$ as the first and the second number 


\section{WANG \& SA}

Table 5. Power performance for test $\rho_{0}=0.00(n=30, \alpha=0.01)$

\begin{tabular}{|c|c|c|c|c|c|c|c|c|c|c|c|c|c|c|c|c|}
\hline \multirow[b]{2}{*}{ b } & \multirow[b]{2}{*}{ g } & \multicolumn{3}{|c|}{$\mathrm{ra}=0.0$} & \multicolumn{3}{|c|}{$\mathrm{ra}=0.2$} & \multicolumn{3}{|c|}{$\mathrm{ra}=0.4$} & \multicolumn{3}{|c|}{$\mathrm{ra}=0.6$} & \multicolumn{3}{|c|}{$\mathrm{ra}=0.8$} \\
\hline & & $\mathrm{Zf}$ & $\mathrm{Zb}$ & $\mathrm{Zc}$ & Zf & $\mathrm{Zb}$ & Zc & $\mathrm{Zf}$ & $\mathrm{Zb}$ & Zc & $\mathrm{Zf}$ & $\mathrm{Zb}$ & Zc & $Z f$ & $\mathrm{Zb}$ & Zc \\
\hline 0.0 & 0 & 0.0103 & 0.0271 & 0.0202 & 0.1037 & 0.1508 & 0.1316 & 0.4585 & 0.4838 & 0.4451 & 0.9084 & 0.8966 & 0.8489 & 0.9996 & 0.9995 & 0.9871 \\
\hline 0.0 & 0 & & 0.0235 & 0.0161 & & 0.1299 & 0.1095 & & 0.4305 & 0.3908 & & 0.8640 & 0.8044 & & 0.9991 & 0.9797 \\
\hline 0.0 & 1 & 0.0102 & 0.0319 & 0.0180 & 0.1046 & 0.1572 & 0.1185 & 0.4646 & 0.5000 & 0.4061 & 0.9090 & 0.9038 & 0.7928 & 0.9996 & 0.9995 & 0.9313 \\
\hline 0.0 & 1 & & 0.0283 & 0.0137 & & 0.1348 & 0.0962 & & 0.4451 & 0.3492 & & 0.8728 & 0.7404 & & 0.9990 & 0.9136 \\
\hline 0.0 & 10 & 0.0147 & 0.0605 & 0.0087 & 0.1247 & 0.2016 & 0.0717 & 0.4893 & 0.5508 & 0.2901 & 0.9128 & 0.9203 & 0.6149 & 0.9997 & 0.9988 & 0.7141 \\
\hline 0.0 & 10 & & 0.0586 & 0.0061 & & 0.1801 & 0.0525 & & 0.4962 & 0.2281 & & 0.8931 & 0.5508 & & 0.9983 & 0.6823 \\
\hline 0.5 & 0 & 0.0114 & 0.0226 & 0.0180 & 0.1074 & 0.1410 & 0.1245 & 0.4594 & 0.4744 & 0.4353 & 0.9026 & 0.8925 & 0.8371 & 0.9996 & 0.9992 & 0.9753 \\
\hline 0.5 & 0 & & 0.0192 & 0.0140 & & 0.1208 & 0.1030 & & 0.4210 & 0.3814 & & 0.8597 & 0.7936 & & 0.9984 & 0.9647 \\
\hline 1.0 & 0 & 0.0129 & 0.0169 & 0.0130 & 0.1051 & 0.0961 & 0.0850 & 0.4051 & 0.3352 & 0.3022 & 0.8197 & 0.7457 & 0.6739 & 0.9929 & 0.9834 & 0.9398 \\
\hline 1.0 & 0 & & 0.0144 & 0.0101 & & 0.0794 & 0.0682 & & 0.2824 & 0.2524 & & 0.6781 & 0.6026 & & 0.9732 & 0.9098 \\
\hline 0.5 & 1 & 0.0115 & 0.0286 & 0.0172 & 0.1047 & 0.1482 & 0.1134 & 0.4621 & 0.4886 & 0.4020 & 0.9055 & 0.8997 & 0.7877 & 0.9995 & 0.9990 & 0.9279 \\
\hline 0.5 & 1 & & 0.0249 & 0.0130 & & 0.1256 & 0.0913 & & 0.4331 & 0.3453 & & 0.8665 & 0.7364 & & 0.9986 & 0.9107 \\
\hline 1.2 & 4 & 0.0148 & 0.0338 & 0.0110 & 0.1203 & 0.1512 & 0.0831 & 0.4721 & 0.4806 & 0.3217 & 0.9012 & 0.8896 & 0.6826 & 0.9996 & 0.9974 & 0.8191 \\
\hline 1.2 & 4 & & 0.0315 & 0.0082 & & 0.1301 & 0.0637 & & 0.4247 & 0.2662 & & 0.8544 & 0.6223 & & 0.9961 & 0.7932 \\
\hline 1.2 & 10 & 0.0163 & 0.0544 & 0.0076 & 0.1310 & 0.1918 & 0.0676 & 0.4912 & 0.5330 & 0.2835 & 0.9086 & 0.9087 & 0.6116 & 0.9997 & 0.9980 & 0.7199 \\
\hline 1.2 & 10 & & 0.0527 & 0.0053 & & 0.1706 & 0.0496 & & 0.4801 & 0.2235 & & 0.8782 & 0.5471 & & 0.9972 & 0.6891 \\
\hline 1.2 & 25 & 0.0215 & 0.0744 & 0.0047 & 0.1591 & 0.2325 & 0.0478 & 0.5077 & 0.5639 & 0.2222 & 0.8869 & 0.8971 & 0.5045 & 0.9989 & 0.9977 & 0.6111 \\
\hline 1.2 & 25 & & 0.0733 & 0.0032 & & 0.2128 & 0.0316 & & 0.5155 & 0.1640 & & 0.8670 & 0.4369 & & 0.9965 & 0.5767 \\
\hline-1.2 & 4 & 0.0145 & 0.0328 & 0.0104 & 0.1208 & 0.1503 & 0.0818 & 0.4723 & 0.4783 & 0.3207 & 0.9022 & 0.8890 & 0.6833 & 0.9996 & 0.9973 & 0.8200 \\
\hline-1.2 & 4 & & 0.0303 & 0.0076 & & 0.1300 & 0.0630 & & 0.4228 & 0.2649 & & 0.8541 & 0.6231 & & 0.9960 & 0.7939 \\
\hline
\end{tabular}

4 Note: $\beta$ : skewness; $\gamma$ : kurtosis; the "Zf", "Zb", and "Zc" results are calculated using the critical points $z_{\alpha}$ and $t_{\alpha}$ as the first and the second number 


\section{A NEW TEST FOR CORRELATION}

1 Table 5 (continued). Power performance for test $\rho_{0}=0.00(n=30, \alpha=0.01)$

\begin{tabular}{|c|c|c|c|c|c|c|c|c|c|c|c|c|c|c|c|c|}
\hline \multirow[b]{2}{*}{ b } & \multirow[b]{2}{*}{ g } & \multicolumn{3}{|c|}{$\mathrm{ra}=0.0$} & \multicolumn{3}{|c|}{$\mathrm{ra}=0.2$} & \multicolumn{3}{|c|}{$\mathrm{ra}=0.4$} & \multicolumn{3}{|c|}{$\mathrm{ra}=0.6$} & \multicolumn{3}{|c|}{$\mathrm{ra}=0.8$} \\
\hline & & $\mathrm{Zf}$ & $\mathrm{Zb}$ & $\mathrm{Zc}$ & Zf & $\mathrm{Zb}$ & Zc & $\mathrm{Zf}$ & $\mathrm{Zb}$ & Zc & Zf & $\mathrm{Zb}$ & Zc & $Z f$ & $\mathrm{Zb}$ & Zc \\
\hline-1.2 & 10 & 0.0162 & 0.0538 & 0.0077 & 0.1289 & 0.1910 & 0.0665 & 0.4912 & 0.5327 & 0.2842 & 0.9081 & 0.9079 & 0.6091 & 0.9997 & 0.9980 & 0.7179 \\
\hline-1.2 & 10 & & 0.0521 & 0.0053 & & 0.1695 & 0.0482 & & 0.4787 & 0.2262 & & 0.8785 & 0.5449 & & 0.9971 & 0.6858 \\
\hline-1.2 & 25 & 0.0211 & 0.0747 & 0.0048 & 0.1578 & 0.2318 & 0.0479 & 0.5102 & 0.5645 & 0.2215 & 0.8888 & 0.8984 & 0.5067 & 0.9988 & 0.9977 & 0.6081 \\
\hline-1.2 & 25 & & 0.0740 & 0.0033 & & 0.2124 & 0.0316 & & 0.5174 & 0.1647 & & 0.8695 & 0.4397 & & 0.9964 & 0.5752 \\
\hline 1.2 & 4 & 0.0156 & 0.0447 & 0.0094 & 0.1249 & 0.1740 & 0.0759 & 0.4840 & 0.5143 & 0.3082 & 0.9113 & 0.9057 & 0.6532 & 0.9998 & 0.9980 & 0.7733 \\
\hline 1.2 & 10 & & 0.0424 & 0.0065 & & 0.1522 & 0.0568 & & 0.4598 & 0.2487 & & 0.8761 & 0.5924 & & 0.9972 & 0.7447 \\
\hline 1.2 & 4 & 0.0160 & 0.0586 & 0.0065 & 0.1348 & 0.2032 & 0.0643 & 0.5128 & 0.5580 & 0.2840 & 0.9298 & 0.9304 & 0.6169 & 1.0000 & 0.9994 & 0.6999 \\
\hline 1.2 & 25 & & 0.0572 & 0.0044 & & 0.1807 & 0.0452 & & 0.5023 & 0.2219 & & 0.9057 & 0.5564 & & 0.9991 & 0.6684 \\
\hline-1.2 & 4 & 0.0142 & 0.0456 & 0.0089 & 0.1245 & 0.1738 & 0.0757 & 0.4854 & 0.5144 & 0.3067 & 0.9111 & 0.9063 & 0.6515 & 0.9998 & 0.9978 & 0.7704 \\
\hline-1.2 & 10 & & 0.0433 & 0.0063 & & 0.1513 & 0.0560 & & 0.4588 & 0.2477 & & 0.8759 & 0.5899 & & 0.9969 & 0.7406 \\
\hline-1.2 & 4 & 0.0157 & 0.0583 & 0.0062 & 0.1342 & 0.2019 & 0.0627 & 0.5101 & 0.5586 & 0.2853 & 0.9308 & 0.9322 & 0.6170 & 1.0000 & 0.9993 & 0.7001 \\
\hline-1.2 & 25 & & 0.0567 & 0.0043 & & 0.1801 & 0.0443 & & 0.5040 & 0.2234 & & 0.9074 & 0.5554 & & 0.9991 & 0.6682 \\
\hline 1.2 & 4 & 0.0207 & 0.0411 & 0.0050 & 0.1453 & 0.1614 & 0.0501 & 0.4815 & 0.4620 & 0.2219 & 0.8882 & 0.8708 & 0.5494 & 0.9991 & 0.9963 & 0.6948 \\
\hline 3.0 & 14 & & 0.0392 & 0.0034 & & 0.1429 & 0.0350 & & 0.4091 & 0.1699 & & 0.8296 & 0.4796 & & 0.9946 & 0.6610 \\
\hline 1.2 & 4 & 0.0186 & 0.0501 & 0.0056 & 0.1423 & 0.1796 & 0.0551 & 0.5095 & 0.5184 & 0.2633 & 0.9139 & 0.9031 & 0.5867 & 0.9999 & 0.9986 & 0.6977 \\
\hline 3.0 & 25 & & 0.0483 & 0.0036 & & 0.1599 & 0.0384 & & 0.4641 & 0.2049 & & 0.8712 & 0.5225 & & 0.9978 & 0.6663 \\
\hline 1.2 & 14 & 0.0216 & 0.0661 & 0.0053 & 0.1507 & 0.2115 & 0.0514 & 0.5075 & 0.5454 & 0.2422 & 0.8979 & 0.8984 & 0.5391 & 0.9995 & 0.9978 & 0.6459 \\
\hline 3.0 & 25 & & 0.0646 & 0.0036 & & 0.1920 & 0.0348 & & 0.4955 & 0.1849 & & 0.8684 & 0.4746 & & 0.9969 & 0.6133 \\
\hline-1.2 & 4 & 0.0194 & 0.0409 & 0.0052 & 0.1433 & 0.1614 & 0.0486 & 0.4814 & 0.4626 & 0.2221 & 0.8886 & 0.8722 & 0.5495 & 0.9991 & 0.9965 & 0.6940 \\
\hline-3.0 & 14 & & 0.0393 & 0.0035 & & 0.1428 & 0.0340 & & 0.4106 & 0.1699 & & 0.8318 & 0.4814 & & 0.9947 & 0.6609 \\
\hline
\end{tabular}

4 Note: $\beta$ : skewness; $\gamma$ : kurtosis; the "Zf", "Zb", and "Zc" results are calculated using the critical points $z_{\alpha}$ and $t_{\alpha}$ as the first and the second number 


\section{WANG \& SA}

1

2 Table 5 (continued). Power performance for test $\rho_{0}=0.00(n=30, \alpha=0.01)$

\begin{tabular}{|c|c|c|c|c|c|c|c|c|c|c|c|c|c|c|c|c|}
\hline \multirow[b]{2}{*}{ b } & \multirow[b]{2}{*}{ g } & \multicolumn{3}{|c|}{$\mathrm{ra}=0.0$} & \multicolumn{3}{|c|}{$\mathrm{ra}=0.2$} & \multicolumn{3}{|c|}{$\mathrm{ra}=0.4$} & \multicolumn{3}{|c|}{$\mathrm{ra}=0.6$} & \multicolumn{3}{|c|}{$\mathrm{ra}=0.8$} \\
\hline & & $\mathrm{Zf}$ & $\mathrm{Zb}$ & $\mathrm{Zc}$ & Zf & $\mathrm{Zb}$ & Zc & $\mathrm{Zf}$ & $\mathrm{Zb}$ & Zc & Zf & $\mathrm{Zb}$ & Zc & Zf & $\mathrm{Zb}$ & Zc \\
\hline-1.2 & 4 & 0.0192 & 0.0501 & 0.0055 & 0.1434 & 0.1806 & 0.0550 & 0.5090 & 0.5181 & 0.2614 & 0.9142 & 0.9036 & 0.5878 & 0.9999 & 0.9983 & 0.6962 \\
\hline-3.0 & 25 & & 0.0483 & 0.0036 & & 0.1600 & 0.0385 & & 0.4644 & 0.2026 & & 0.8726 & 0.5256 & & 0.9976 & 0.6636 \\
\hline-1.2 & 14 & 0.0216 & 0.0659 & 0.0053 & 0.1499 & 0.2138 & 0.0515 & 0.5060 & 0.5448 & 0.2406 & 0.9001 & 0.9015 & 0.5416 & 0.9994 & 0.9978 & 0.6478 \\
\hline-3.0 & 25 & & 0.0648 & 0.0035 & & 0.1941 & 0.0358 & & 0.4956 & 0.1838 & & 0.8722 & 0.4775 & & 0.9968 & 0.6163 \\
\hline 3.0 & 25 & 0.0277 & 0.0645 & 0.0040 & 0.1702 & 0.2099 & 0.0431 & 0.5080 & 0.5167 & 0.2028 & 0.8724 & 0.8581 & 0.4780 & 0.9978 & 0.9945 & 0.6159 \\
\hline 3.0 & 25 & & 0.0632 & 0.0025 & & 0.1929 & 0.0293 & & 0.4710 & 0.1516 & & 0.8239 & 0.4133 & & 0.9922 & 0.5829 \\
\hline-3.0 & 25 & 0.0282 & 0.0646 & 0.0044 & 0.1719 & 0.2110 & 0.0424 & 0.5079 & 0.5176 & 0.2031 & 0.8715 & 0.8589 & 0.4801 & 0.9978 & 0.9946 & 0.6142 \\
\hline-3.0 & 25 & & 0.0633 & 0.0028 & & 0.1938 & 0.0280 & & 0.4734 & 0.1533 & & 0.8239 & 0.4151 & & 0.9923 & 0.5815 \\
\hline
\end{tabular}

$5 \quad$ Note: $\beta$ : skewness; $\gamma$ : kurtosis; the "Zf", "Zb", and "Zc" results are calculated using the critical points $z_{\alpha}$ and $t_{\alpha}$ as the first and the second number 
A NEW TEST FOR CORRELATION

1 Table 6 Power performance for test $\rho_{0}=0.55(n=30, \alpha=0.10)$

\begin{tabular}{|c|c|c|c|c|c|c|c|c|c|c|c|}
\hline \multirow[b]{2}{*}{$\beta$} & \multirow[b]{2}{*}{$Y$} & \multirow[b]{2}{*}{ CP } & \multicolumn{3}{|c|}{$\rho_{a}=0.55$} & \multicolumn{3}{|c|}{$\rho_{\alpha}=0.60$} & \multicolumn{3}{|c|}{$\rho_{\alpha}=0.70$} \\
\hline & & & $Z f$ & $\mathbf{Z b}$ & Zc & $\mathrm{Zf}$ & $\mathrm{Zb}$ & Zc & $\mathrm{Zf}$ & $\mathrm{Zb}$ & Zc \\
\hline 0.0 & 0 & $z_{\alpha}$ & 0.0991 & 0.1275 & 0.1139 & 0.3005 & 0.3038 & 0.2861 & 0.6538 & 0.4908 & 0.4779 \\
\hline 0.0 & 0 & $t_{\alpha}$ & & 0.1115 & 0.0978 & & 0.2632 & 0.2452 & & 0.4201 & 0.4073 \\
\hline 0.0 & 1 & $z_{\alpha}$ & 0.1017 & 0.1352 & 0.1142 & 0.2990 & 0.3064 & 0.2795 & 0.6526 & 0.4850 & 0.4678 \\
\hline 0.0 & 1 & $t_{\alpha}$ & & 0.1219 & 0.0999 & & 0.2729 & 0.2452 & & 0.4226 & 0.4043 \\
\hline 0.0 & 10 & $Z_{\alpha}$ & 0.1483 & 0.1923 & 0.1358 & 0.3445 & 0.3497 & 0.2934 & 0.6604 & 0.4896 & 0.4611 \\
\hline 0.0 & 10 & $t_{\alpha}$ & & 0.1855 & 0.1253 & & 0.3296 & 0.2690 & & 0.4502 & 0.4190 \\
\hline 0.5 & 0 & $Z_{\alpha}$ & 0.1075 & 0.1312 & 0.1164 & 0.3058 & 0.3037 & 0.2853 & 0.6496 & 0.4919 & 0.4789 \\
\hline 0.5 & 0 & $t_{\alpha}$ & & 0.1153 & 0.1005 & & 0.2662 & 0.2475 & & 0.4245 & 0.4114 \\
\hline 1.0 & 0 & $Z_{\alpha}$ & 0.0914 & 0.1044 & 0.0918 & 0.2330 & 0.2350 & 0.2156 & 0.4929 & 0.4230 & 0.4030 \\
\hline 1.0 & 0 & $t_{\alpha}$ & & 0.0938 & 0.0813 & & 0.2095 & 0.1901 & & 0.3756 & 0.3561 \\
\hline 0.5 & 1 & $Z_{\alpha}$ & 0.1046 & 0.1355 & 0.1142 & 0.3026 & 0.3062 & 0.2798 & 0.6517 & 0.4870 & 0.4698 \\
\hline 0.5 & 1 & $t_{\alpha}$ & & 0.1220 & 0.0997 & & 0.2717 & 0.2443 & & 0.4259 & 0.4082 \\
\hline 1.2 & 4 & $Z_{\alpha}$ & 0.1280 & 0.1656 & 0.1259 & 0.3246 & 0.3255 & 0.2843 & 0.6491 & 0.4795 & 0.4556 \\
\hline 1.2 & 4 & $t_{\alpha}$ & & 0.1556 & 0.1143 & & 0.3011 & 0.2573 & & 0.4313 & 0.4057 \\
\hline 1.2 & 10 & $Z_{\alpha}$ & 0.1508 & 0.1968 & 0.1387 & 0.3491 & 0.3548 & 0.2969 & 0.6560 & 0.4845 & 0.4546 \\
\hline 1.2 & 10 & $t_{\alpha}$ & & 0.1889 & 0.1274 & & 0.3348 & 0.2727 & & 0.4466 & 0.4144 \\
\hline 1.2 & 25 & $Z_{\alpha}$ & 0.2114 & 0.2388 & 0.1621 & 0.3977 & 0.3716 & 0.3024 & 0.6554 & 0.4797 & 0.4407 \\
\hline 1.2 & 25 & $t_{\alpha}$ & & 0.2341 & 0.1523 & & 0.3571 & 0.2827 & & 0.4537 & 0.4114 \\
\hline
\end{tabular}

Note: $\beta$ : skewness; $\gamma$ : kurtosis; CP: Critical Point 


\section{WANG \& SA}

1 Table 6 (continued). Power performance for test $\rho_{0}=0.55(n=30, \alpha=0.10)$

\begin{tabular}{|c|c|c|c|c|c|c|c|c|c|c|c|}
\hline \multirow[b]{2}{*}{$\beta$} & \multirow[b]{2}{*}{$Y$} & \multirow[b]{2}{*}{ CP } & \multicolumn{3}{|c|}{$\alpha=0.10$} & \multicolumn{3}{|c|}{$\alpha=0.05$} & \multicolumn{3}{|c|}{$\alpha=0.01$} \\
\hline & & & $\mathrm{Zf}$ & $\mathrm{Zb}$ & $\mathrm{Zc}$ & Zf & $\mathrm{Zb}$ & Zc & $\mathrm{Zf}$ & $\mathrm{Zb}$ & Zc \\
\hline-1.2 & 4 & $z_{\alpha}$ & 0.1295 & 0.1656 & 0.1266 & 0.3213 & 0.3262 & 0.2836 & 0.6487 & 0.4781 & 0.4545 \\
\hline-1.2 & 4 & $t_{\alpha}$ & & 0.1558 & 0.1148 & & 0.2998 & 0.2550 & & 0.4299 & 0.4046 \\
\hline-1.2 & 10 & $Z_{\alpha}$ & 0.1517 & 0.1972 & 0.1394 & 0.3468 & 0.3545 & 0.2967 & 0.6554 & 0.4886 & 0.4593 \\
\hline-1.2 & 10 & $t_{\alpha}$ & & 0.1899 & 0.1284 & & 0.3342 & 0.2726 & & 0.4492 & 0.4176 \\
\hline-1.2 & 25 & $z_{\alpha}$ & 0.2116 & 0.2375 & 0.1610 & 0.3954 & 0.3696 & 0.2989 & 0.6558 & 0.4755 & 0.4379 \\
\hline-1.2 & 25 & $t_{\alpha}$ & & 0.2328 & 0.1509 & & 0.3566 & 0.2803 & & 0.4509 & 0.4097 \\
\hline 1.2 & 4 & $Z \alpha$ & 0.1359 & 0.1958 & 0.1373 & 0.3357 & 0.3711 & 0.3103 & 0.6653 & 0.5225 & 0.4913 \\
\hline 1.2 & 10 & $t_{\alpha}$ & & 0.1872 & 0.1255 & & 0.3491 & 0.2842 & & 0.4801 & 0.4465 \\
\hline 1.2 & 4 & $Z \alpha$ & 0.1600 & 0.2497 & 0.1594 & 0.3745 & 0.4387 & 0.3492 & 0.7149 & 0.5731 & 0.5348 \\
\hline 1.2 & 25 & $t_{\alpha}$ & & 0.2448 & 0.1480 & & 0.4216 & 0.3255 & & 0.5386 & 0.4972 \\
\hline-1.2 & 4 & $Z \alpha$ & 0.1358 & 0.1953 & 0.1373 & 0.3366 & 0.3704 & 0.3095 & 0.6645 & 0.5211 & 0.4904 \\
\hline-1.2 & 10 & $t_{\alpha}$ & & 0.1879 & 0.1266 & & 0.3486 & 0.2838 & & 0.4789 & 0.4458 \\
\hline-1.2 & 4 & $Z \alpha$ & 0.1570 & 0.2459 & 0.1557 & 0.3746 & 0.4401 & 0.3518 & 0.7164 & 0.5735 & 0.5343 \\
\hline-1.2 & 25 & $t_{\alpha}$ & & 0.2412 & 0.1447 & & 0.4239 & 0.3287 & & 0.5383 & 0.4955 \\
\hline 1.2 & 4 & $Z \alpha$ & 0.1553 & 0.1993 & 0.1414 & 0.3418 & 0.3569 & 0.3005 & 0.6380 & 0.4908 & 0.4662 \\
\hline 3.0 & 14 & $t_{\alpha}$ & & 0.1937 & 0.1320 & & 0.3395 & 0.2798 & & 0.4554 & 0.4288 \\
\hline 1.2 & 4 & $z \alpha$ & 0.1724 & 0.2435 & 0.1626 & 0.3790 & 0.4097 & 0.3344 & 0.6958 & 0.5346 & 0.5011 \\
\hline 3.0 & 25 & $t_{\alpha}$ & & 0.2378 & 0.1515 & & 0.3927 & 0.3116 & & 0.5009 & 0.4644 \\
\hline
\end{tabular}

$4 \quad$ Note: $\beta$ : skewness; $\gamma$ : kurtosis; CP: Critical Point 


\section{A NEW TEST FOR CORRELATION}

1 Table 6 (continued). Power performance for test $\rho_{0}=0.55(n=30, \alpha=0.10)$

\begin{tabular}{|c|c|c|c|c|c|c|c|c|c|c|c|}
\hline \multirow[b]{2}{*}{$\beta$} & \multirow[b]{2}{*}{$Y$} & \multirow[b]{2}{*}{ CP } & \multicolumn{3}{|c|}{$\alpha=0.10$} & \multicolumn{3}{|c|}{$\alpha=0.05$} & \multicolumn{3}{|c|}{$\alpha=0.01$} \\
\hline & & & $\mathrm{Zf}$ & $\mathrm{Zb}$ & Zc & $\mathrm{Zf}$ & $\mathrm{Zb}$ & $\mathrm{Zc}$ & $Z f$ & $Z b$ & Zc \\
\hline 1.2 & 14 & $Z_{\alpha}$ & 0.1942 & 0.2334 & 0.1578 & 0.3843 & 0.3659 & 0.3004 & 0.6628 & 0.4698 & 0.4376 \\
\hline 3.0 & 25 & $t_{\alpha}$ & & 0.2278 & 0.1479 & & 0.3510 & 0.2806 & & 0.4415 & 0.4064 \\
\hline-1.2 & 4 & $Z_{\alpha}$ & 0.1586 & 0.2051 & 0.1459 & 0.3404 & 0.3566 & 0.3006 & 0.6378 & 0.4939 & 0.4685 \\
\hline-3.0 & 14 & ta & & 0.1990 & 0.1363 & & 0.3395 & 0.2802 & & 0.4584 & 0.4315 \\
\hline-1.2 & 4 & $z_{\alpha}$ & 0.1700 & 0.2413 & 0.1599 & 0.3790 & 0.4073 & 0.3333 & 0.6958 & 0.5321 & 0.4995 \\
\hline-3.0 & 25 & $t_{\alpha}$ & & 0.2365 & 0.1498 & & 0.3901 & 0.3109 & & 0.4983 & 0.4631 \\
\hline-1.2 & 14 & $Z \alpha$ & 0.1955 & 0.2326 & 0.1588 & 0.3865 & 0.3638 & 0.2994 & 0.6620 & 0.4616 & 0.4303 \\
\hline-3.0 & 25 & $t_{\alpha}$ & & 0.2270 & 0.1484 & & 0.3495 & 0.2805 & & 0.4329 & 0.3992 \\
\hline 3.0 & 25 & $Z \alpha$ & 0.2214 & 0.2517 & 0.1699 & 0.3995 & 0.3760 & 0.3003 & 0.6430 & 0.4740 & 0.4292 \\
\hline 3.0 & 25 & $t_{\alpha}$ & & 0.2473 & 0.1604 & & 0.3630 & 0.2821 & & 0.4489 & 0.4009 \\
\hline-3.0 & 25 & $Z \alpha$ & 0.2210 & 0.2514 & 0.1685 & 0.3996 & 0.3776 & 0.3012 & 0.6411 & 0.4731 & 0.4284 \\
\hline-3.0 & 25 & ta & & 0.2464 & 0.1584 & & 0.3644 & 0.2822 & & 0.4480 & 0.400 \\
\hline
\end{tabular}

4 Note: $\beta$ : skewness; $\gamma$ : kurtosis; CP: Critical Point 